\title{
Combined theoretical and experimental study of the valence, Rydberg and ionic states of chlorobenzene
}

Michael H. Palmer, ${ }^{1 \mathrm{a}}$ Trevor Ridley, ${ }^{1, \mathrm{~b}}$ Søren Vrønning Hoffmann, ${ }^{2, \mathrm{~b}}$ Nykola C. Jones, ${ }^{2, b}$ Marcello Coreno, ${ }^{3, b}$ Monica de Simone, ${ }^{4, b}$ Cesare Grazioli, ${ }^{4,5}$ Teng Zhang, ${ }^{6}$ Malgorzata Biczysko, ${ }^{7,8, b}$ Alberto Baiardi, ${ }^{8}$ and Kirk A. Peterson ${ }^{9, b}$

${ }^{1}$ School of Chemistry, University of Edinburgh, Joseph Black Building, David Brewster Road, Edinburgh EH9 3FJ, Scotland, UK

2 ISA, Department of Physics and Astronomy, Aarhus University, Ny Munkegade 120, DK8000 Aarhus C, Denmark

${ }^{3}$ CNR-ISM, Basovizza Area Science Park, 1-34149 Trieste, Italy

${ }^{4}$ CNR-IOM Laboratorio TASC, Trieste, Italy

${ }^{5}$ Department of Chemical and Pharmaceutical Sciences, University of Trieste, Italy

${ }^{6}$ University of Uppsala - Dept. of Physics and Astronomy, Uppsala, Sweden.

${ }^{7}$ National Research Council ICCOM-CNR, UOS di Pisa, Via G. Moruzzi 1, I-56124 Pisa, Italy

${ }^{8}$ Scuola Normale Superiore, Piazza Cavalieri 7, 56126 Pisa, Italy

${ }^{9}$ Washington State University, Department of Chemistry, Pullman, WA 99164-4630, USA

a) Email: m.h.palmer@ed.ac.uk: Telephone: +44 (0) 1316504765

b) Electronic addresses: tr01@staffmail.ed.ac.uk; vronning@phys.au.dk; nykj@phys.au.dk; marcello.coreno@elettra.eu; desimone@iom.cnr.it;_malgorzata.biczysko@sns.it; kipeters@wsu.edu

\begin{abstract}
New photoelectron (PE) and ultra violet (UV) and vacuum UV (VUV) spectra have been obtained for chlorobenzene by synchrotron study with higher sensitivity and resolution than previous work and are subjected to detailed analysis. In addition, we report on the massresolved $(2+1)$ resonance enhanced multiphoton ionization (REMPI) spectra of a jet-cooled sample. Both the VUV and REMPI spectra have enabled identification of a considerable number of Rydberg states for the first time. The use of $a b$ initio calculations which include
\end{abstract}


both multi-reference multi-root doubles and singles configuration interaction (MRD-CI) and time dependent density functional theoretical (TDDFT) methods, has led to major advances in interpretation of the vibrational structure of the ionic and electronically excited states. FranckCondon (FC) analyses of the PE spectra, including both hot and cold bands, indicate much more complex envelopes than previously thought. The sequence of ionic states can be best interpreted by our multi-configuration self-consistent field (MCSCF) computations, and also by comparison of the calculated vibrational structure of the $\mathrm{B}$ and $\mathrm{C}$ ionic states with experiment; these conclusions suggest that the leading sequence is the same as that of iodobenzene and bromobenzene, namely: $\mathrm{X}^{2} \mathrm{~B}_{1}\left(3 \mathrm{~b}_{1}{ }^{-1}\right)<\mathrm{A}^{2} \mathrm{~A}_{2}\left(1 \mathrm{a}_{2}^{-1}\right)<\mathrm{B}^{2} \mathrm{~B}_{2}\left(6 \mathrm{~b}_{2}{ }^{-1}\right)<\mathrm{C}^{2} \mathrm{~B}_{1}\left(2 \mathrm{~b}_{1}{ }^{-}\right.$ ${ }^{1}$ ). The onset near $4.6 \mathrm{eV}$ has been investigated using MRD-CI and TDDFT calculations; the principal component of this band is ${ }^{1} \mathrm{~B}_{2}$ and an interpretation based on the superposition of FC and Herzberg-Teller contributions has been performed. The other low-lying absorption band near $5.8 \mathrm{eV}$ is dominated by a ${ }^{1} \mathrm{~A}_{1}$ state, but an underlying weak ${ }^{1} \mathrm{~B}_{1}$ state $\left(\pi \sigma^{*}\right)$ is also found. The strongest band in the VUV spectrum near $6.7 \mathrm{eV}$ is poorly resolved, and is analyzed in terms of two $\pi \pi^{*}$ states of ${ }^{1} \mathrm{~A}_{1}$ (higher oscillator strength) and ${ }^{1} \mathrm{~B}_{2}$ (lower oscillator strength) symmetries, respectively. The calculated vertical excitation energies of these two states is critically dependent upon the presence of Rydberg functions in the basis set, since both manifolds are strongly perturbed by the Rydberg states in this energy range. A number of equilibrium structures of the ionic and singlet excited states show that the molecular structure is less subject to variation than corresponding studies for iodobenzene and bromobenzene. 


\section{INTRODUCTION}

In this Paper, we present new high-resolution, room temperature synchrotron-excited photoelectron spectra (PES) and 1-photon, ultraviolet (UV) and vacuum ultraviolet (VUV) absorption spectra of chlorobenzene $(\boldsymbol{P h} \boldsymbol{C l})$. In addition, we report on the mass-resolved $(2+$ 1) resonance enhanced multiphoton ionization (REMPI) spectra of a jet-cooled sample which provide additional information about the Rydberg states in particular. The current VUV energy range for $\boldsymbol{P h} \boldsymbol{C l}$ is up to approximately $11 \mathrm{eV}$, and Rydberg states leading to the first four ionic state band systems will occur in this energy range. In the current Paper the FranckCondon (FC) vibrational profiles of both hot and cold bands of the ionized states are analyzed in detail for the first time, putting the knowledge of these states on a more rigorous footing. Similarly, the onset of the $\boldsymbol{P h} \boldsymbol{C l}$ absorption spectrum (the $\mathrm{S}_{1}$ state) cannot be analyzed by FC terms alone, and Herzberg-Teller (HT) terms make a substantial contribution. The analyses of the spectra are supported by ab initio configuration interaction $(\mathrm{CI})$ and time dependent density functional theory (TDDFT) calculations of both vertical and adiabatic ionization and excitation energies.

The new study builds on three recent papers $^{1-3}$ where we reported PES and VUV studies for iodobenzene $(\boldsymbol{P h I})$ and bromobenzene $(\boldsymbol{P h B r})$. These two spectral techniques are synergistic, since the vibronic structure observed in the PES is widely used as a fingerprint to identify the ionic core of the observed Rydberg state or series in the absorption spectra. ${ }^{4-10}$ As expected, the vibrational profiles exhibited in both types of spectra become increasingly similar for high- $n$ Rydberg states; for low- $n$ states relatively minor perturbations can be expected. For these PES and VUV spectral comparisons, detailed analysis of the FC effects is essential, and this is carried out in the present study. 
Since $\boldsymbol{P h C l}$ is a high profile molecule with an extensive literature, we concentrate on leading references for earlier work; additional relatively less relevant sources and information are given within these references. The most accurate investigations of the lower ionic states have been by 1-photon VUV laser-excited mass-analyzed threshold ionization (MATI) spectroscopy. ${ }^{11}$ A more wide-ranging survey of the PES is by Potts et al. ${ }^{12}$ but our new PES and VUV spectra for $\boldsymbol{P h C l}$ both enhance the sensitivity and resolution of the conventional $\mathrm{PES}^{12}$ spectral information, while our theoretical analysis improves the level of spectral interpretation of both methods. ${ }^{11,12}$ This PES + VUV analysis leads to the identification of a considerable number of new Rydberg states, where we exploit our earlier techniques for simplifying the observed VUV spectra, by removal of broad structures, leaving the sharp Rydberg features more evident. In order to facilitate comparison with earlier work, we use eV, $\mathrm{nm}$ and $\mathrm{cm}^{-1}$ for PES, UV and VUV spectra, while only $\mathrm{cm}^{-1}$ are used for Rydberg states.

The most accurate value for the first ionization energy, $\mathrm{IE}_{1}$, (X state) for $\boldsymbol{P h} \boldsymbol{C l}$ is 73 172.1(1.6) $\mathrm{cm}^{-1}(9.0722(2) \mathrm{eV})$ which was obtained from an experiment equivalent to MATI in which electrons rather than ions were collected. ${ }^{13}$ The MATI spectrum ${ }^{11}$ gave the energy of $\mathrm{IE}_{3}$ (B state) as $91404(5) \mathrm{cm}^{-1}\left(11.3327(7) \mathrm{eV}\right.$ ), but no values for either the $\mathrm{IE}_{2}$ (A state) or $\mathrm{IE}_{4}$ (C state). These other values (of importance to the current study) were obtained by Potts et al. ${ }^{12}$ using synchrotron- and HeI-excited PES, as $\left.\mathrm{IE}_{2}, 78292 \mathrm{~cm}^{-1}(9.707) \mathrm{eV}\right)$ and $\mathrm{IE}_{4}, 94358$ $\mathrm{cm}^{-1}(11.699 \mathrm{eV})$; these first four IEs can be expected to generate Rydberg states in the current VUV range, (e.g. a 3s state with an $\mathrm{IE}_{3}$ ionic core near $8.6 \mathrm{eV}$ ), but those based on higher IEs can be omitted from the current study.

Although our IE measurements are broadly in agreement with those reported, ${ }^{12}$ our calculations of the relevant Franck-Condon factors (FCFs) show that the spectra are certainly more complex than previously reported, with many of the observed peaks having additional vibrational components, on both leading and trailing energy sides. The calculated FCFs for 
the ground and second excited state of the ion will also be compared with the intensities of the bands in the MATI spectrum, and this leads to additional assignments. ${ }^{11}$

The first three bands in the absorption spectrum of $\boldsymbol{P h C l}$ are well-known, ${ }^{14}$ with maxima near 4.5, 5.8 and $6.8 \mathrm{eV}$; these are usually denoted as $\mathrm{S}_{1-3}$. The $\mathrm{S}_{1-3}$ bands are largely valence in nature and closely resemble the principal singlet transitions in benzene $(4.86,6.20$ and $6.97 \mathrm{eV}$ ). Since spin-orbit coupling (SOC) in $\boldsymbol{P h B r}$ has been shown to be negligible, at least in the FC region, ${ }^{15}$ we assume that the same is true in $\mathbf{P h C l}$ and hence it is appropriate to use the terms singlet and triplet for the electronic states.

Although there have been an extensive number of photochemical studies of gas phase $\boldsymbol{P h} \boldsymbol{C l}$ with excitation into the $\mathrm{S}_{1}$ state ${ }^{16-18}$ and the $\mathrm{S}_{2}$ state, ${ }^{16,19,20}$ most of these studies are more concerned with dynamics and do not impact on the current study. However, some of these photochemical studies $^{21,22}$ calculate potential energy surfaces for the low-lying excited singlet and triplet states which are discussed in the relevant sections below, since they relate to our adiabatic excitation energies (AEE).

The first VUV study of the $\boldsymbol{P h} \boldsymbol{C l}$ absorption spectrum (up to $11.27 \mathrm{eV}$ ) was neither measured nor analyzed in detail, ${ }^{23}$ possibly since the UV region was found to be very similar to benzene itself, but shifted to longer wavelengths. ${ }^{14,23,24}$ Kimura et al. ${ }^{25,26}$ fitted the low energy region with four Gaussian bands (denoted as A to D), with maxima at 4.71, 5.95, 6.72 and $6.88 \mathrm{eV}$, respectively. Their theoretical analysis, using only $\pi$-MOs on $\mathrm{C}$ and $\mathrm{Cl}$ atoms, was correspondingly limited to $\pi \pi^{*}$ valence and charge-transfer $(\mathrm{C}-\mathrm{T})$ states; this led to symmetry assignments of $A$ to $D$ as $B_{2}, A_{1}, B_{2}$ and $A_{1}$ states respectively, corresponding to the $\mathrm{B}_{2 \mathrm{u}}, \mathrm{B}_{1 \mathrm{u}}$ and $\mathrm{E}_{1 \mathrm{u}}$ states of benzene. ${ }^{25,26}$ Robin, ${ }^{24}$ concluded that the lowest 'nonbenzenoid' bands in $\boldsymbol{P h} \boldsymbol{C l}$ (and also $\boldsymbol{P h F}$ ) are $\pi \sigma^{*}$ transitions.

No positive assignments of Rydberg states were made in these VUV absorption studies of $\boldsymbol{P h} \boldsymbol{C l}$. A strong band at $146 \mathrm{~nm}$ was thought to relate to similar bands in alkyl 
chlorides, ${ }^{23}$ but the VUV spectrum shows several discrete bands between 145 and $165 \mathrm{~nm} ;{ }^{23}$ we assign these below to Rydberg bands. Later, Asselin et al. ${ }^{27}$ using double resonance MPIPES (DR-MPI-PES) via the $S_{1}$ state, observed three Rydberg states which were assigned as 3d, 4p and 4d states, with origins at 60300,64690 and $66390 \mathrm{~cm}^{-1}$.

Our new analysis of the VUV profile, as well as the PES, assists in observing numerous Rydberg states in the current $\boldsymbol{P h} \boldsymbol{C l}$ spectrum. In particular, we use the vibronic structure observed in the PES as a fingerprint to identify the ionic core of the observed Rydberg state or series. Characteristic quantum defects are used to further assign a considerable variety of s, p, d and f Rydberg states. In addition, many more Rydberg states are observed in the higher-resolution REMPI spectra, and polarization data unambiguously identifies the symmetry of many of these.

Our computational procedures below include multi-configuration SCF (MCSCF) studies of the lower ionic (X, A to C) states, TDDFT studies of the AEEs of some singlet states, and multi-reference singles and doubles CI studies (MRD-CI) of the overall vertical pattern of UV and VUV absorption. In addition, equation-of-motion-ionization-potentialcoupled cluster singles and doubles (EOMIP-CCSD) methods were applied to the ionic and $\mathrm{S}_{1}$ neutral states.

\section{EXPERIMENTAL AND COMPUTATIONAL PROCEDURES}

\section{A. Experimental}

The PES for $\boldsymbol{P h} \boldsymbol{C l}$ (CAS Registry Number 108-90-7, Sigma-Aldrich) were measured at the Gas Phase Photoemission beamline ${ }^{28}$ at the Elettra synchrotron radiation laboratory in Trieste, Italy. A detailed description of the experiment is given in an earlier Paper. ${ }^{1}$ 
For data acquired with a photon energy of $30 \mathrm{eV}$ over the binding energy range 8.1$12.8 \mathrm{eV}$ (stepsize $1 \mathrm{meV}$ ), the overall experimental energy resolution was $\sim 8 \mathrm{meV}$. We estimate our experimental uncertainties in the absolute energies and the energy separations in a particular band system to be approximately \pm 2 and $\pm 0.5 \mathrm{meV}$, respectively. The low-energy region containing the five lowest $I E s, \mathrm{IE}_{1-5}$, is shown in Fig. 1(a); only the first four are relevant to the VUV spectral analysis. The values of $\mathrm{IE}_{2}$ and $\mathrm{IE}_{4}$, shown in Table I, along with all other PES peaks were measured by fitting the data points to sets of Gaussian functions; we accept the values previously determined ${ }^{11,13}$ for $\mathrm{IE}_{1}$ and $\mathrm{IE}_{3}$. A wide scan spectrum was recorded for the range 8-25 eV using $95 \mathrm{eV}$ photons with an overall experimental energy resolution of $25 \mathrm{meV}$, and its theoretical correlation is deferred to the supplementary material. $^{29}$

The UV and VUV absorption spectrum for $\boldsymbol{P h C l}$ was measured at the ASTRID2 storage ring at Aarhus University, Denmark, using the recently modified AU-UV beam line which is described in detail in a previous Paper. ${ }^{2}$ The spectrum was measured in the energy range from $3.757 \mathrm{eV}\left(330 \mathrm{~nm}, 30302 \mathrm{~cm}^{-1}\right)$ to $10.781 \mathrm{eV}\left(115 \mathrm{~nm}, 86954 \mathrm{~cm}^{-1}\right)$ using data points separated by $0.05 \mathrm{~nm}$ ( 4 to $40 \mathrm{~cm}^{-1}$ in this energy range) and is shown in Fig. 1(b) and Fig. 2.

The mass-resolved $(2+1)$ REMPI spectra of jet-cooled $\boldsymbol{P h C l}$ were measured at the University of Edinburgh. The molecular beam was generated by pulsing a mixture of $\boldsymbol{P h} \boldsymbol{C l}$ at its vapor pressure with an atmosphere of He through a General Valve pulsed nozzle, with a $250 \mu \mathrm{m}$ diameter aperture, into the ionization region of a linear time-of-flight mass spectrometer. Ions were collected at $90^{\circ}$ to both the molecular and laser beams. Mass-resolved ion signals were processed by a Stanford Research SR250 boxcar integrator and stored on a PC. 
Radiation was provided by a dye laser (Lambda Physik FL3002) pumped by a XeCl excimer laser (Lambda Physik EMG201MSC). For the $(2+1)$ REMPI spectra, the frequency doubled outputs of $\mathrm{C} 153, \mathrm{R} 6 \mathrm{G}$, and RB dyes were used that had laser pulse energies of 2-5 mJ after frequency doubling. The laser radiation was focused by a $6 \mathrm{~cm}$ focal length lens into the interaction region with the molecular beam. Circular polarization was achieved by passing the beam through a Soleil Babinet prism.

Because of the high power intensities required to observe the high- $n$ Rydberg states, the dominant fragments in most cases were $\mathrm{C}^{+}$(the most common fragment) and $\mathrm{C}_{2}^{+}$. However, since many atomic resonances were also detected by collecting $\mathrm{C}^{+}$, thus complicating the spectrum, $\mathrm{C}_{2}{ }^{+}$was usually collected. The fragmentation, following the 2- or 3-photon absorption to the Rydberg states and 1-photon ionization, probably occurs by absorption of one or more additional photons to the repulsive ionic states. For simplicity, however, all spectra recorded in the present work will be denoted as $(2+1)$ REMPI spectra. Spectra were normalized to the square of the simultaneously recorded laser power. For dye fundamental wavelengths, calibration was obtained from the $\mathrm{I}_{2}(\mathrm{~B} \leftarrow \mathrm{X})$ fluorescence excitation spectrum. Although the calibration was accurate to $\pm 1 \mathrm{~cm}^{-1}$, the final uncertainties in the reported band positions are mainly dictated by their widths and may be up to $\pm 10 \mathrm{~cm}^{-1}$.

\section{B. Computational methods and basis sets}

Equilibrium structures, AEEs and harmonic frequencies for several singlet states ${ }^{30-32}$ were obtained using the TDDFT method in GAUSSIAN-09. ${ }^{33}$ In addition, coupled cluster methods implemented in the CFOUR program package, ${ }^{34}$ were used to obtain equilibrium geometries and harmonic vibrational frequencies; coupled cluster singles and doubles (CCSD) for the neutral ground state and EOMIP-CCSD for the first four electronic states of the cation 
$\left(1^{2} \mathrm{~B}_{1}, 1^{2} \mathrm{~A}_{2}, 1^{2} \mathrm{~B}_{2}\right.$, and $\left.2^{2} \mathrm{~B}_{1}\right){ }^{35}$ State-averaged (SA) MCSCF methods, as implemented in MOLPRO ${ }^{36}$ were used for some higher ionic states; SA-MCSCF uses restricted Hartree-Fock (RHF) methods. Full details of ionic and singlet state equilibrium structures are given in the supplementary material. ${ }^{29}$ Valence shell numbering is used to enable comparisons with the other $\boldsymbol{P h} \boldsymbol{X}$ molecules. In these studies, these $\mathrm{C}_{2 \mathrm{v}}$ molecules lie in the yz-plane, where the zaxis lies along the $\mathrm{C}_{2}$ axis.

Vibrational FC profiles ${ }^{37-40}$ within the Adiabatic Hessian approximation (FCAH), were generated from both the UHF and RHF wave-functions. Only a few AEEs were determined, since root swapping, where many states have rather similar structures and energies, prevented the success of the computational focus on particular states; below we show all states where significant oscillator strength was found. Vertical excitation energies (VEE) for valence states, determined by an MRD-CI method, ${ }^{41-43}$ as implemented in GAMESS-UK, ${ }^{44}$ were also utilized.

In all these CCSD and MCSCF calculations, the frozen core approximation was invoked, whereby the $1 \mathrm{~s}$ electrons of $\mathrm{C}$ and the $1 \mathrm{~s}, 2 \mathrm{~s}$ and $2 \mathrm{p}$ electrons of $\mathrm{Cl}$ were not correlated. In the MCSCF calculations of both ionic and valence states, core and inner valence orbitals were frozen to $2 \mathrm{e}$ occupancy (total $23 \mathrm{MOs})$ leading to 14 active orbitals $\left(3 \mathrm{a}_{1}+5 \mathrm{~b}_{1}+\right.$ $\left.3 b_{2}+3 a_{2}\right)$; the $X^{1} A_{1}$ state generated 414361 determinants, while the ionic states generated greater than $1.5 \times 10^{6}$ determinants in each symmetry. In the MRD-CI study, more fully described in our previous studies, ${ }^{8-10}$ only the 11 core orbitals $\left(7 a_{1}+1 b_{1}+3 b_{2}\right)$ were frozen at 2e occupancy; the full valence shell occupied orbitals $\left(8 a_{1}+3 b_{1}+6 b_{2}+1 a_{2}\right)$ were included in this method, together with up to 120 virtual MOs (VMOs).

The main part of the theoretical study used the augmented correlation-consistent polarized triple zeta valence basis set (aug-cc-pVTZ). ${ }^{45-47}$ In the MRD-CI study, the lack of relatively diffuse functions in this basis set did not lead to acceptable energies for the onset 
region of the UV and VUV spectrum. A quadruple zeta valence with (single) polarization (QZVP) extended version of the earlier triple zeta valence + polarization (TZVP) basis set was used; ${ }^{48,49}$ the lowest exponents of each TZVP type on each centre were repeated with $1 / 3$ of the exponent value; this generates a more diffuse basis and also increases the number of valence shell VMOs relative to polarization functions in aug-cc-pVTZ. We calculated the positions of low-lying Rydberg states, several of which were not detected in the VUV analysis below, by use of sets of s-, p-, d- and f-Rydberg functions, which followed our previous procedure. ${ }^{8-10}$ In addition, anharmonic corrections to frequencies were computed by means of the generalized vibrational perturbation theory $(\text { GVPT2 })^{50}$ using the B3LYP functional in conjunction with the double- $\zeta$ SNSD basis set, ${ }^{51}$ this was previously successful for a series of halogenated organic compounds. ${ }^{52}$

\section{RESULTS AND DISCUSSION}

\section{A. Aspects of the PES of relevance to the VUV absorption spectrum}

The lowest group of ionization energies relevant to Rydberg state presence in the present VUV spectrum is shown in Fig. 1(a). The $\mathrm{X}^{2} \mathrm{~B}_{1}$ and $\mathrm{A}^{2} \mathrm{~A}_{2}$ states are derived from the splitting of the degenerate ${ }^{2} \mathrm{E}_{1 \mathrm{~g}}$ benzene state by the halogen atom. It is clear that the PES baseline is not recovered between these two states, and hence vibronic coupling seems inevitable. The $\mathrm{B}$ and $\mathrm{C}$ states (attributed to the $\mathrm{B}^{2} \mathrm{~B}_{2}$ and $\mathrm{C}^{2} \mathrm{~B}_{1}$ states below), both largely result from the removal of in-plane (lone pair) $\sigma$ - and $\pi$-electrons respectively on the halogen. ${ }^{12}$ The appearance of the first three of these are very similar to those in the PES of $\boldsymbol{P h B r},{ }^{3}$ namely, the first contains extended, sharp vibrational structure, the second contains only a few very broad peaks, while the last contains sharp peaks with a very intense origin 
and very little vibrational structure. As was the case with $\boldsymbol{P h} \boldsymbol{B r}$, this results in Rydberg states with a B state ionic core standing out in the VUV spectrum, e.g. the 3s Rydberg state labelled in Fig. 1(b). This is the band highlighted as an intense peak in an earlier VUV absorption spectrum. ${ }^{23}$ The $\mathrm{C}$ state band of $\boldsymbol{P h C l}$ contains only two broad peaks and differs from those of $\boldsymbol{P h B r}$ and $\boldsymbol{P h I}$ which contain appreciable vibrational structure. Again, the width of the peaks of the $\mathrm{C}$ state are markedly broader than those for the B state, and again this suggests vibronic coupling of the higher of this pair of states, similar to that of the $\mathrm{X}$ and A states.

The four ionization processes (low to high IE) correspond to leading electron configurations with loss of a single electron from each of the HOMO to HOMO-3 shown in Fig. 3; for convenience we include the lowest unoccupied MOs in Fig. 3 which are relevant to the singlet state discussion below. At the single configuration level, and in agreement with most previous work, ${ }^{12}$ the four HOMO to HOMO-3 states are of $\pi<\pi<\sigma<\pi$ symmetry. When ionization occurs, the energy ordering is maintained and thus, overall, we obtain the ionic state sequence $\mathrm{X}^{2} \mathrm{~B}_{1}\left(3 \mathrm{~b}_{1}{ }^{-1}\right)<\mathrm{A}^{2} \mathrm{~A}_{2}\left(1 \mathrm{a}_{2}{ }^{-1}\right)<\mathrm{B}^{2} \mathrm{~B}_{2}\left(6 \mathrm{~b}_{2}^{-1}\right)<\mathrm{C}^{2} \mathrm{~B}_{1}\left(2 \mathrm{~b}_{1}{ }^{-1}\right)$ (Table I), which agrees with previous equilibrium structure calculations, ${ }^{53}$ and this is discussed again in more detail below. The electron density in the lowest four ionic states is only close to that of ${ }^{2} \mathrm{E}_{1 \mathrm{~g}}$ (IE 1 ) in benzene for the $1 \mathrm{a}_{2}^{-1}$ ionization ( $\left.\mathrm{IE}_{2}\right)$. In $\boldsymbol{P h} \boldsymbol{X}$, the corresponding positions for $\mathrm{IE}_{\mathrm{V}}$ in the $1 \mathrm{a}_{2}$ band are $9.504(\mathrm{I}), 9.654(\mathrm{Br}), 9.633(\mathrm{Cl})$ and $9.802(\mathrm{~F}) \mathrm{eV}$, showing the second order effect of the nodal halogen. However, for $\boldsymbol{P h F}$, the splitting of $1 \mathrm{a}_{2}^{-1}\left(\mathrm{IE}_{2}\right)$ from the lowest adiabatic (A) vibrational band of $3 b_{1}{ }^{-1}\left(\mathrm{IE}_{1}\right)$ vertical $(\mathrm{V})$ ionization is poorly defined. The $\boldsymbol{P h} \boldsymbol{X}$ series show that $\mathrm{IE}_{1}$ and $\mathrm{IE}_{2}$ are separated by $0.75,0.66,0.56$ and $0.60 \mathrm{eV}$ for $\boldsymbol{P h I} \boldsymbol{P} \boldsymbol{h} \boldsymbol{B r}$, $\boldsymbol{P h} \boldsymbol{C l}$ and $\boldsymbol{P h F}$ respectively; indicating that the separation of the corresponding Rydberg states can be expected to reduce across the halogen series. We will discuss briefly below how $\mathrm{IE}_{\mathrm{V}}$ and $\mathrm{IE}_{\mathrm{A}}$ of the $\mathrm{A}^{2} \mathrm{~A}_{2}$ states of $\boldsymbol{P} \boldsymbol{h} \boldsymbol{X}$ are related. 


\section{B. Detailed analysis of the PES}

\section{Vibrational analysis of the PES spectrum}

The PES profiles of $\mathrm{IE}_{1-4}$ are comparable with those recorded earlier with $\mathrm{HeI}(21.2$ eV) irradiation, ${ }^{12}$ but show much higher count rates. The measured peak positions, their separations from the electronic origins and their assignments based on the FCF calculations are shown in Table II. A Table of the calculated frequencies of the vibrations in the ionic states principally observed in the PES, together with the calculated squares of the transition dipole moment integrals, is given in the supplementary material. ${ }^{29}$

Table III shows the vibrational modes of $\boldsymbol{P h} \boldsymbol{C l}$ numbered according to the Mulliken convention. The anharmonic frequencies from EOMIP calculations were calculated using the mode-specific scaling procedure based on the Duschinsky matrix ${ }^{38}$ the procedure is described in detail in our previous studies. ${ }^{1,3}$ As was observed for $\boldsymbol{P h I}$ and $\boldsymbol{P h} \boldsymbol{B r}$, the anharmonic frequencies calculated by this method are approximately $2 \%$ higher than the experimental values.

In our previous simulations of the PES of $\boldsymbol{P h I ^ { 1 }}$ and $\boldsymbol{P h} \boldsymbol{B r}{ }^{3}$, we found it necessary to use different full-width, half-maximum (FWHM) bandwidths for different ionic state simulations; we will abbreviate this to bandwidth for the remainder of this Paper. Typical values for the $\mathrm{X}^{2} \mathrm{~B}_{1}, \mathrm{~A}^{2} \mathrm{~A}_{2}$ and $\mathrm{B}^{2} \mathrm{~B}_{2}$ states of $\boldsymbol{P h I}$ were 150,300 and $400 \mathrm{~cm}^{-1}$ respectively. We initially assumed that the different bandwidths required were a consequence of the states having different lifetimes where internal conversion (IC) to a lower occurs on the fs timescale.

However, this simple procedure did not produce an acceptable fit of the experimental spectrum of $\boldsymbol{P h B r}$, since there is a build-up of intensity in the energy region between the $\mathrm{X}^{2} \mathrm{~B}_{1}$ and $\mathrm{A}^{2} \mathrm{~A}_{2}$ states which we could not reproduce. This was attributed to vibronic coupling 
between the two states analogous to that observed in an ab initio study between the equivalent states of $\boldsymbol{P h F}{ }^{58,59}$ Although we did not directly include vibronic coupling in our simulations, the fact that we had to use different optimized bandwidths for the simulations of different states showed that we had gone some way to account for its effect, at least on an empirical basis. From a comparison of our simulations with both the conventional PES and MATI experimental spectra, we proposed that $\mathrm{a}_{2}$ vibrational mode, $v_{23}$, is most effective in inducing this vibronic coupling. We investigate whether vibronic coupling also occurs in $\boldsymbol{P h C l}$ from the simulations of the vibrational profiles of the PES bands described below.

a. The $X^{2} B_{1}$ band. The $\mathrm{X}^{2} \mathrm{~B}_{1}$ band of the PES of $\boldsymbol{P h C l}$ and a stick spectrum of the largest calculated line intensities of the cold bands are shown in Figs. 4(b) and 4(a), respectively. The 1-photon MATI spectrum, ${ }^{11}$ with intensity scaled to match the PES origin

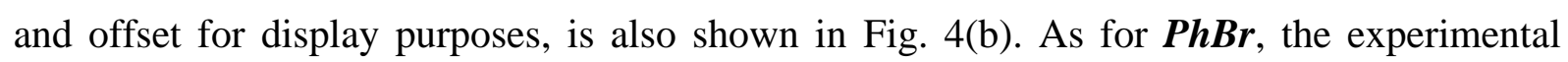
spectra are dominated by progressions of $v_{11}\left(\sim 420 \mathrm{~cm}^{-1}\right)$, with $v=0-2$, built on the electronic origin, and also on one quantum of $v_{7}$ (calculated frequency $1138 \mathrm{~cm}^{-1}$ ) and one quantum of $v_{4}\left(1639 \mathrm{~cm}^{-1}\right)$.

The MATI spectrum is much simpler than the PES because of the vibrational and rotational cooling of the sample by the molecular beam and the narrow linewidth of the excitation laser $\left(\sim 1 \mathrm{~cm}^{-1}\right)$. It can be seen that the observed band intensities in the MATI spectrum are very similar to those in the conventional PES. Since the intensities in both spectra are largely determined by FC overlap with the ground state of the neutral molecule this is to be expected.

The MATI technique involves exciting long-lived ( $>10 \mu \mathrm{s})$ Rydberg states that lie a few wavenumbers below the ionization limit and then ionizing them using a delayed pulsed electric field. Thus, any process that decreases the lifetime of these Rydberg states will 
decrease the detection efficiency, and hence the MATI intensities, and this may explain any differences in intensities in the PES and MATI spectra.

The MATI spectrum also provides more accurate $a_{1}$ vibrational frequencies that can be compared with our calculated values. The frequencies of the vibrations observed with the greatest intensity are presented in Table III. In addition, many vibrations of other symmetries were observed by Asselin et al., ${ }^{56}$ using REMPI with photoelectron detection, and these frequencies are also compared with the calculated values in Table III.

The simulation of the total $\mathrm{X}^{2} \mathrm{~B}_{1}$ PES band obtained by convoluting the full stick spectrum, including hot bands, (shown in Fig. 4(a)) with a bandwidth of $150 \mathrm{~cm}^{-1}$ is shown in Fig. 4(b). The simulation has been shifted in energy to superimpose the experimental spectrum. The simulation is a good reproduction of the experimental spectrum in two respects, namely the number of vibrational modes that give rise to bands with appreciable intensity and their frequencies. In addition, the relative intensities of the individual members of the $v_{11}$ progressions are good.

As was the case for $\boldsymbol{P h I}$ and $\boldsymbol{P h} \boldsymbol{B r}$, each peak in the PES is notably asymmetric, having a tail to low energy as can be seen from an expansion of the origin band shown in the inset of Fig. 4(a). This is due to the inclusion of several unresolved sequence bands in the observed envelope and, in order to carry out a rigorous simulation of the total PES, it is essential to include all hot bands in our FCF calculations. A stick spectrum of the calculated wavenumber shifts of the sequence bands associated with the $\mathrm{X}^{2} \mathrm{~B}_{1}$ electronic origin band is shown in the inset of Fig. 4(a). The first, $n=1$, member of each of the most intense $v_{\mathrm{N}}$ sequence band series, $\mathrm{N}^{1}$ is labelled; $n>1$ members are denoted by color-coded asterisks.

A convolution of the stick spectrum with a bandwidth of $150 \mathrm{~cm}^{-1}$ is shown in the red trace and this gives a good reproduction of the contour of the experimental peak. All three spectra share a common base line, i.e. there is a positive count at $-250 \mathrm{~cm}^{-1}$. Assuming that 
neither the origin band itself nor its associated sequence bands are broadened by any form of interaction and that the resolution of the spectrometer is $\sim 70 \mathrm{~cm}^{-1}$, this bandwidth yields an unperturbed band contour for $\boldsymbol{P h} \boldsymbol{C l}$ that is $\sim 80 \mathrm{~cm}^{-1}$ wide, the same as for $\boldsymbol{P h I}$ and $\boldsymbol{P h} \boldsymbol{B r}$.

It can be seen that the maxima of the experimental peak and its optimized simulation (73 $\left.138 \mathrm{~cm}^{-1}\right)$ are offset from the origin of the stick spectrum $\left(73168 \mathrm{~cm}^{-1}\right)$ as a result of the convolution. This offset is confirmed by the fact that the position of the origin of the stick spectrum is in very good agreement that derived from the MATI spectrum ${ }^{11}\left(73177(5) \mathrm{cm}^{-1}\right)$, shown in Fig. 4(b), and well within our experimental uncertainty $\left( \pm 16 \mathrm{~cm}^{-1}\right)$.

The overall simulation increasingly deviates from the experimental PES with increasing energy, starting around $74500 \mathrm{~cm}^{-1}$, and our simulations cannot directly account for the observed intensity in the region leading up to the $\mathrm{A}^{2} \mathrm{~A}_{2}$ state. In our previous study of the equivalent spectrum of $\mathbf{P h B r},{ }^{3}$ we proposed that this build-up of intensity is due to coupling between the two states by a similar mechanism to that described by Baldea et al. ${ }^{58,59}$ in an ab initio study on the five lowest ionic states of $\boldsymbol{P h F}$. They proposed that the $\mathrm{X}^{2} \mathrm{~B}_{1}$ and $\mathrm{A}^{2} \mathrm{~A}_{2}$ states were strongly coupled by a $\mathrm{b}_{2}$ vibration which they labelled $v_{25}\left(v_{23}\right.$ in the Mulliken convention used here). Experimentally, one consequence of the coupling was that a vibronically induced component was introduced into the PES built on one quantum of this vibration. This results in a build-up of intensity, consisting of $A_{2}$ vibronic levels, between the origins of the two ionic states which increases as the $\mathrm{A}^{2} \mathrm{~A}_{2}$ state origin, and the conical intersection between the two potential surfaces, is approached. We propose that the same coupling is taking place in $\boldsymbol{P h} \boldsymbol{C l}$.

In $\boldsymbol{P h B r}$ we concluded that an $\mathrm{X}^{2} \mathrm{~B}_{1}$ state $\mathrm{b}_{2}$ vibrational mode with an experimental frequency of $1402 \mathrm{~cm}^{-1}$ (calculated frequency $1410 \mathrm{~cm}^{-1}$ ) induces the strongest coupling with the $\mathrm{A}^{2} \mathrm{~A}_{2}$ state. The Duschinsky matrix for the transition showed that the major component of the vibration is mode $v_{23}$ in the neutral ground state, the same one that was cited for the 
equivalent coupling in $\boldsymbol{P h F}{ }^{58,59}$ The Duschinsky matrix also showed that $v_{23}$ is unique in being the only one whose frequency is greatly reduced from the ground state to the ionic state (calculated frequencies 1618 and $1402 \mathrm{~cm}^{-1}$, respectively); a similar decrease occurs in $\boldsymbol{P h C l}$ (calculated frequencies 1623 and $1410 \mathrm{~cm}^{-1}$ ).

Two consequences of the coupling are that the $23^{1} 11^{n}$ bands were observed in the MATI spectrum and that the PES was more intense than the optimized simulation at these energies. Consequently, we assign the three bands indicated by black '+'s in Fig. 4(b) as $23^{1}$, $23^{1} 11^{1}$ and $23^{1} 11^{2}$. Since, in $\mathbf{P h C l}, \mathrm{X}^{1} \mathrm{~A}_{1}$ mode $v_{23}$ also makes a significant contribution to $\mathrm{X}^{2} \mathrm{~B}_{1}$ mode $v_{24}$ (calculated frequency $1554 \mathrm{~cm}^{-1}$ ), we further assign the three bands indicated by red '+'s in Fig. 4(b) as $24^{1}, 24^{1} 11^{1}$ and $24^{1} 11^{2}$.

The present identification of the vibronically induced bands in the MATI spectrum necessitates reassignments of what is given in the literature. ${ }^{11}$ Both $v_{23}$ and $v_{24}$ fundamental bands were reasonably assigned to $\mathrm{a}_{1}$ vibrations from a comparison with their neutral ground state frequencies, and the expectation that bands involving $\mathrm{a}_{1}$ vibrations will usually be intense. However, our calculated FCFs show that only a few $a_{1}$ vibrational bands have high intensity and that vibronic coupling has to be invoked to fully assign the MATI spectrum.

Finally, many vibrations of $\mathrm{a}_{1}, \mathrm{a}_{2}$ and $\mathrm{b}_{2}$ symmetries were observed by Asselin et al., ${ }^{56}$ using REMPI with photoelectron detection. These frequencies are compared with the calculated values in Table III and the agreement between our calculated frequencies and their observed $a_{1}, a_{2}$ and $b_{2}$ frequencies are very good. The agreement for the $b_{1}$ frequencies is also good if a minor reassignment is made where three ionic state frequencies are labelled $v_{16-18}$ rather than $v_{15-17}$

b. The $A^{2} A_{2}$ band. The identification of the A state as the $\mathrm{A}^{2} \mathrm{~A}_{2}$ band is not in dispute and is shown in Fig. 5(b). As in the equivalent band in the PES of the other three 
$\boldsymbol{P h} \boldsymbol{X}$ molecules, the peaks are much broader than those of the $\mathrm{X}^{2} \mathrm{~B}_{1}$ state and only two peaks can be identified (see Table II). Our calculations indicate that six vibrational modes, $v_{11}, v_{10}$, $v_{9}, v_{8}, v_{7}$ and $v_{4}$ give rise to bands with significant intensity as shown in Fig. 5(a).

As was the case for $\boldsymbol{P h I}$, it was necessary to increase the bandwidth in the simulation to $\sim 600 \mathrm{~cm}^{-1}$ to achieve even an approximate match of the experimental spectrum. However, as for $\boldsymbol{P h B r}$, we are still unable to generate an acceptable simulation; two examples are shown Fig. 5(b) in which the intensities of the origins in both simulations are matched with the experimental origin but, the baselines are set to zero counts (green trace) and the experimental 'baseline' at $77500 \mathrm{~cm}^{-1}$ (red trace). Specifically, we cannot reproduce either the large background around $77500 \mathrm{~cm}^{-1}$ or the relative intensity of the origin band $\left(\sim 78500 \mathrm{~cm}^{-1}\right)$ to that of the unresolved fundamental bands $\left(\sim 79500 \mathrm{~cm}^{-1}\right)$.

In general, our simulations of the PES of the monohalobenzenes are unable to reproduce the observed intensity in the energy region between the $X^{2} B_{1}$ and $A^{2} A_{2}$ bands, e.g. in the $\boldsymbol{P h C l}$ spectrum our combined simulations predict near zero intensity at $77500 \mathrm{~cm}^{-1}$, whereas there is a significant signal at that energy as can be seen from Fig. 5(b). As this intensity is a consequence of the vibronic coupling between these two states described above and we cannot account for such vibronic coupling in our ionic state calculations; this sets a limitation to our simulation procedures.

Qualitatively, the appearance of the $\mathrm{X}^{2} \mathrm{~B}_{1}$ and $\mathrm{A}^{2} \mathrm{~A}_{2}$ PES bands of $\boldsymbol{P h} \boldsymbol{C l}$ and $\boldsymbol{P h} \boldsymbol{F}$ are very similar and we believe that the states are similarly coupled in both molecules. We believe that the results of ab initio study of the vibronic coupling between the two states ${ }^{58,59}$ for $\boldsymbol{P h F}$ solve some but not all of these issues. We will give the results of our analysis of the $\mathrm{X}^{2} \mathrm{~B}_{1}$ and $\mathrm{A}^{2} \mathrm{~A}_{2}$ PES bands of $\boldsymbol{P h} \boldsymbol{F}$ in a future paper. ${ }^{60}$ For the present, we propose that the most intense peak of the $\mathrm{A}^{2} \mathrm{~A}_{2}$ band does not correspond to the adiabatic IE, but to a maximum in the superimposed vibronically induced $\mathrm{X}^{2} \mathrm{~B}_{1}$ vibrational bands and electronically 
allowed $\mathrm{A}^{2} \mathrm{~A}_{2}$ bands. Consequently, the most intense peak $\left(9.712 \mathrm{eV}, 78327 \mathrm{~cm}^{-1}\right)$ of the of the $\mathrm{A}^{2} \mathrm{~A}_{2}$ state of $\boldsymbol{P h} \boldsymbol{C l}$ also does not correspond to the adiabatic IE, as has been previously assumed. ${ }^{12}$ We believe that the adiabatic IE $\mathrm{A}^{2} \mathrm{~A}_{2}$ for $\boldsymbol{P h} \boldsymbol{F}$ is not observed in the current spectra and probably lies $\sim 1000 \mathrm{~cm}^{-1}$ lower in energy.

c. The B and C bands. The symmetry assignments of the $\mathrm{B}$ and $\mathrm{C}$ states for $\boldsymbol{P h} \boldsymbol{C l}$ have proved controversial, and call for some comment here. The B ionic states of both $\boldsymbol{P h I}$ and $\boldsymbol{P h B r}$ definitely have ${ }^{2} \mathrm{~B}_{2}$ symmetry, ${ }^{1,3}$ and several other studies have also supported this conclusion with an IE energy ordering of $1^{2} \mathrm{~B}_{2}<2^{2} \mathrm{~B}_{1}$ states of $\boldsymbol{P h C l}$; these views include B state similarities ${ }^{12}$ in the PES between $\boldsymbol{P h C l}$ and $\boldsymbol{P h B r}$, and long lifetimes $(>10 \mu \mathrm{s})$ in a collision cell. ${ }^{61}$ Penning ionization electron spectroscopy (PIES), in which a metastable atom $\left(\mathrm{He}^{*}\left(2^{3} \mathrm{~S}\right)\right)$ impact causes ionization, has also been used to support the $\mathrm{IE}_{3,4}$ sequence as above for all four $\boldsymbol{P h X}$ molecules. ${ }^{62}$ Equilibrium structure calculations of AEEs for the $\boldsymbol{P h} \boldsymbol{C l}$ cations, ${ }^{53,61,63}$ using CASPT2 or CASSCF methods gave a sequence ${ }^{53}$ for the AEE $\left(\mathrm{T}_{0}\right)$ as $1^{2} \mathrm{~B}_{1}<1^{2} \mathrm{~A}_{2}<1^{2} \mathrm{~B}_{2}<2^{2} \mathrm{~B}_{1}<1^{2} \mathrm{~A}_{1}<2^{2} \mathrm{~B}_{2}<3^{2} \mathrm{~B}_{1}$. Subsequent CASPT2 calculations predicted the same AEE $\left(\mathrm{T}_{0}\right)$ sequence, but the optimized geometries of three states $\left(1^{2} \mathrm{~B}_{2}, 2^{2} \mathrm{~B}_{1}\right.$, and $1^{2} \mathrm{~A}_{1}$ ) were significantly different ${ }^{63}$ from previously reported CASSCF optimized geometries.

In a major criticism, the results of photo-induced Rydberg ionization (PIRI) ${ }^{64,65}$ and resonance enhanced multiphoton dissociation (REMPD) ${ }^{65,66}$ studies appear to contradict the above $\mathrm{B}$ and $\mathrm{C}$ state assignments. In the PIRI experiment, a vibronic level of the $\mathrm{X}^{2} \mathrm{~B}_{1}$ ionic state is initially populated and 1-photon transitions to the upper $\left(\mathrm{B}, \mathrm{IE}_{3}\right)$ state are monitored. The lowest energy transition in the PIRI spectrum excited from the origin of the $\mathrm{X}^{2} \mathrm{~B}_{1}$ state to the B state was observed at $18219 \mathrm{~cm}^{-1}$ and corresponds to an excited state level at 91 391(10) $\mathrm{cm}^{-1}$ using an $\mathrm{IE}_{1}$ of $73172 \mathrm{~cm}^{-1} .{ }^{13}$ This level was assigned ${ }^{64,65}$ to the $\mathrm{B}$ state origin 
which, if correct, means that the state must have ${ }^{2} \mathrm{~B}_{1}$ symmetry since 1-photon transitions from a ${ }^{2} \mathrm{~B}_{1}$ state to ${ }^{2} \mathrm{~B}_{2}$ state are electronically forbidden. We discuss this issue further below.

Our contribution to the nature of the B and $\mathrm{C}$ states in $\boldsymbol{P h C l}$ involves stick spectra of the largest calculated line intensities of the cold bands in the PES of the ${ }^{2} \mathrm{~B}_{2}$ and $2^{2} \mathrm{~B}_{1}$ states which are shown in Figs. 6(a) and 7(a), respectively. A simulation of the B state PES band obtained by convoluting the full ${ }^{2} \mathrm{~B}_{2}$ stick spectrum, including hot bands, with a bandwidth of $150 \mathrm{~cm}^{-1}$ is shown in Fig. 6(b). Unlike the $\mathrm{X}^{2} \mathrm{~B}_{1}$ state, the electronic origin derived from the simulation coincides with the observed band maximum $\left(91366 \mathrm{~cm}^{-1}\right)$ since there is significant sequence band structure to both high and low wavenumber of it. The origin obtained from the MATI spectrum $\left(91404 \mathrm{~cm}^{-1}\right)^{11}$ does not lie within our estimated experimental uncertainty $\left( \pm 16 \mathrm{~cm}^{-1}\right)$ and we have no explanation for this.

The simulation shown in Fig. 6(b) gives a reasonable fit of the observed B state PES band, but does not give as good a fit as was observed for the equivalent state of $\boldsymbol{P h} \boldsymbol{B r} .^{3}$ In particular, the calculated intensity of the $10^{1}$ band is significantly greater than is observed experimentally. The $10^{1}$ band, although observable, is also very weak in the MATI spectrum. However, the calculated line intensities for the $2^{2} \mathrm{~B}_{1}$ state shown in Fig. 7(a), where the relative intensity of the $10^{1}$ band is even greater, generate an unacceptable simulation of the $\mathrm{B}$ state PES band.

The fact that the $\mathrm{C}$ state bands are broadened (see below) suggests that the $\mathrm{B}$ and $\mathrm{C}$ states, as in $\boldsymbol{P h F}$, are coupled and this could result in some bands appearing in the B state spectrum as a result of vibronic coupling, as observed in the $\mathrm{X}^{2} \mathrm{~B}_{1}$ spectrum, due to coupling with the $\mathrm{A}^{2} \mathrm{~A}_{2}$ state. Bands that arise from vibronic coupling do not appear in our calculated FC profiles. However, the weakness in the current B state simulation is that the calculated $10^{1}$ band intensity is larger than what is observed, the reverse of the effect of vibronic coupling. Therefore, although the discrepancy is probably a consequence of coupling, it cannot be 
attributed to the direct analogue of this effect. Furthermore, the coupling between these two states appears to be quite weak since our $1^{2} B_{2}$ simulation accounts for all of the experimental intensity in the region leading up to the $\mathrm{C}$ state origin.

The calculated harmonic frequencies for $v_{11}$ in the $1^{2} \mathrm{~B}_{2}$ and $2^{2} \mathrm{~B}_{1}$ states are 386 and $343 \mathrm{~cm}^{-1}$, respectively. The former value is in good agreement with the observed value in the B state MATI spectrum of $382 \mathrm{~cm}^{-1}$, which also suggests that the state has ${ }^{2} \mathrm{~B}_{2}$ symmetry. However, our calculations show that $v_{11}$ in the $1^{2} B_{2}$ state is unique among the calculated frequencies of all of the ionic states in having an anharmonic frequency of $412 \mathrm{~cm}^{-1}$; this positive anharmonicity makes the assignment less conclusive.

A full convolution of the $2^{2} \mathrm{~B}_{1}$ state line intensities with a bandwidth of $300 \mathrm{~cm}^{-1}$ gives a good simulation of the origin band of the C state as shown in the insert of Fig. 7(a); from this, a value of $94358 \mathrm{~cm}^{-1}$ is obtained for $\mathrm{IE}_{4}$. However, the simulation does not accurately reproduce the vibrational profile in the observed PES as shown in Fig. 7(b). In particular, as for the B state simulation, the calculated intensity of the $10^{1}$ band is too large.

In summary, we conclude that, even though the simulations of the B and C state PES bands using the calculated $1^{2} \mathrm{~B}_{2}$ and $2^{2} \mathrm{~B}_{1}$ line intensities, respectively, are not as good as those we have obtained for some other PES bands of $\boldsymbol{P h I}$ and $\boldsymbol{P h} \boldsymbol{B r},{ }^{1,3}$ these line intensities provide better fits than those obtained from the reverse assignments. Hence, our current calculations, while not conclusive, all point to the $\mathrm{B}^{2} \mathrm{~B}_{2}$ and $\mathrm{C}^{2} \mathrm{~B}_{1}$ assignments as being most likely.

This conclusion leads to the necessity for another interpretation of the PIRI and REMPD spectra observed following excitation from the origin of the $\mathrm{X}^{2} \mathrm{~B}_{1}$ state; ${ }^{64-66}$ the PIRI spectrum is shown in Fig. 6(b) and has been aligned on the energy scale assuming that all transitions are from the origin. The lowest energy transition to the B state $\left(18219 \mathrm{~cm}^{-1}\right)$ in the PIRI spectrum was assigned ${ }^{64}$ as the $0^{0}$ band, but this cannot be the case as the transition would have $A_{2}$ symmetry and hence be 1-photon forbidden, if the $\mathrm{B}^{2} \mathrm{~B}_{2}$ assignment is correct. 
The same band was observed in the REMPD spectrum and similarly assigned. ${ }^{64}$ In a second REMPD study, ${ }^{66}$ it was assumed that the $\mathrm{B}$ state had ${ }^{2} \mathrm{~B}_{2}$ symmetry and that the same transition was from the $\mathrm{X}^{2} \mathrm{~B}_{1}$ origin to one quantum of an $\mathrm{a}_{2}$ vibrational mode in the $\mathrm{B}$ state. This assignment can be ruled out since the $18219 \mathrm{~cm}^{-1}$ transition terminates in an upper state level of $91391(10) \mathrm{cm}^{-1}$ which is in good agreement with the electronic origin of the B state observed in the PES, $91366(60) \mathrm{cm}^{-1}$, and MATI spectrum, $91404(5) \mathrm{cm}^{-1} .^{11}$

In a future Paper on $\boldsymbol{P h F},{ }^{60}$ we will confirm the conclusions of Walter et al. ${ }^{67}$ that the electronically forbidden $\mathrm{B}^{2} \mathrm{~B}_{2} \leftarrow \mathrm{X}^{2} \mathrm{~B}_{1}$ transitions in the REMPD spectra are induced by one quantum of two $a_{2}$ vibrational modes, $v_{14}$ and $v_{12}$ and that the same assignment applies to the PIRI spectrum. We now use these conclusions to offer the following tentative suggestions to explain the anomalies in the equivalent spectra of $\boldsymbol{P h C l}$.

We propose that both PIRI and REMPD $\mathrm{B}^{2} \mathrm{~B}_{2} \leftarrow \mathrm{X}^{2} \mathrm{~B}_{1}$ transitions of $\boldsymbol{P h} \boldsymbol{C l}$ are induced by one quantum of $v_{14}$ and that the band at $18603 \mathrm{~cm}^{-1}$, which was previously assigned ${ }^{64}$ as $11^{1}$, is the $14^{1}$ band. Using the most accurate IEs for the $\mathrm{X}$ and $\mathrm{B}$ states, means that this transition terminates on a level $375 \mathrm{~cm}^{-1}$ above the origin. This value is consistent with our calculated frequencies for either $v_{11}$ or $v_{14}$ which are very similar, namely 416 and $397 \mathrm{~cm}^{-1}$, respectively.

An alternative assignment for the band that was assigned as the origin, and whose energy is consistent with this, remains problematical. However, we very tentatively assign it as $30_{1}^{2}$ band of the $\mathrm{B}^{2} \mathrm{~B}_{2} \leftarrow \mathrm{X}^{2} \mathrm{~B}_{1}$ transition based on the following observations. First, the transition should have favourable FC overlap as a result of the calculated frequency of the $b_{2}$ mode which is much lower in the $\mathrm{B}^{2} \mathrm{~B}_{2}$ state $\left(155 \mathrm{~cm}^{-1}\right)$ than in the neutral ground state (297 $\left.\mathrm{cm}^{-1}\right)$, the neutral $\mathrm{S}_{1}$ state $\left(291 \mathrm{~cm}^{-1}\right)$ and the ionic ground state $\left(303 \mathrm{~cm}^{-1}\right)$. A similar example of favourable FC overlap is that we calculate appreciable intensity in the $30^{2}$ band in the PES of the $\mathrm{B}^{2} \mathrm{~B}_{2}$ state (see Fig. 6); no other overtone of a non-totally symmetric vibration is 
calculated to have such a large intensity in the PES simulations of any $\boldsymbol{P h} \boldsymbol{X}$ molecule. Second, the $30_{1}^{2}$ band will only be shifted by a few $\mathrm{cm}^{-1}$ from the origin band. Finally, the rotational contours of the $30^{1}$ and origin bands of the ${ }^{1} \mathrm{~B}_{2} \leftarrow \mathrm{X}^{2} \mathrm{~A}_{1}$ neutral transition overlap and therefore the former may have been unintentionally excited in the excitation step of the PIRI process (this could not happen in $\boldsymbol{P h F}$ ). Thus, the $30^{1}$ level of the ${ }^{1} \mathrm{~B}_{2}$ state of the neutral would be populated in the first step and the $30^{1}$ level of the $\mathrm{X}^{2} \mathrm{~B}_{1}$ state of the ion in the second. The $30^{1}$ level of the $\mathrm{X}^{2} \mathrm{~B}_{1}$ state of the ion would always be populated to some extent in the REMPD experiment.

\section{Summary of the simulations of the vibrational profiles of the PES of PhX molecules}

Having generated simulations of the vibrational profiles observed in the PES of $\boldsymbol{P h I}{ }^{1}$ $\boldsymbol{P h} \boldsymbol{B r}^{2}$ and $\boldsymbol{P h C l}$, we are now in a position to present a summary of the strengths and limitations of our procedures and the results are summarized in Table IV; the estimated accuracies are qualitative, and based on inspection only. The main conclusion to be derived from the data is that the accuracy of the simulation of a particular state is largely dependent on the degree of inter-state coupling that it undergoes since our procedures cannot account for this. In general, for a pair of strongly coupled states, we cannot reproduce the vibrational structure of the upper state or the build-up of intensity between them. The most identifiable factor that influences the strength of the coupling is the separation of the IEs (shown in Table IV) and in some cases these vary significantly from molecule to molecule, the most marked being that between $\mathrm{IE}_{2}$ and $\mathrm{IE}_{3}$.

Our simulations of the structured part of the $\mathrm{IE}_{1}$ bands for all three molecules is very good. The only minor discrepancy is in the presence of some bands in the high-energy region of the structured experimental spectrum which appear to arise from vibronic coupling with 
$\mathrm{IE}_{2}$; since these IEs are well separated, such bands are weak. However, as the ionization energy approaches $\mathrm{IE}_{2}$, the intensity of these bands also increases resulting in a build-up of a featureless background, which we attribute to the increasing strength of the coupling. Thus, the accuracy of our simulations for this region and for the $\mathrm{IE}_{2}$ band itself is generally poor. As the separation of $\mathrm{IE}_{1}$ and $\mathrm{IE}_{2}$ decreases with decreasing halogen mass, the simulation of the $\mathrm{IE}_{2}$ band of $\boldsymbol{P h I}$ is slightly better than that of $\boldsymbol{P h} \boldsymbol{C l}$.

Since the energy of $\mathrm{IE}_{3}$ varies most significantly with halogen mass, the simulation of its PES band provides the best illustration of the effect of coupling on the accuracy of the simulation. Thus, in $\boldsymbol{P h I} \mathrm{IE}_{3}$ lies quite close to $\mathrm{IE}_{2}$, and these couple (and consequently also indirectly with $\mathrm{IE}_{1}$ ); even though this PES band is comprized of only three very broad peaks, these are not well reproduced by the calculated profiles; ${ }^{1}$ this contrasts with $\boldsymbol{P h B r}$ where $\mathrm{IE}_{3}$ is isolated, and its spectrum is very accurately reproduced. ${ }^{3}$ As shown in Fig. 6, the $\mathrm{IE}_{3}$ band in $\boldsymbol{P h C l}$ is less well reproduced, and this is probably because it is slightly coupled to $\mathrm{IE}_{4}$. The same coupling means that the simulation of $\mathrm{IE}_{4}$ in $\mathbf{P h C l}$ is not as good as it is for the other two molecules. We will discuss the effects of vibronic coupling on the PES of all four $\boldsymbol{P h} \boldsymbol{X}$ molecules in detail in a future Paper. ${ }^{60}$

\section{Detailed analysis of the UV and VUV absorption spectrum}

\section{Overall aspects of the $U V$ and VUV absorption spectrum of PhCl and its theoretical interpretation}

Our experimental UV and VUV spectrum (Fig. 2) also shows our calculated valence shell VEEs and their oscillator strengths (OSs) calculated with the MRD-CI method. This includes all single and double excitations from all MOs (i.e. including $\pi$ - and $\sigma$-MOs) into the 
active set of VMOs. The full list of VEE state energies, OSs and second moments of the charge distribution (SMCD), both of which evaluate the nature of the transitions as valence or Rydberg, is presented in the supplementary material. ${ }^{29}$ The AEEs of the valence states are shown in Table V, which includes calculated adiabatic (TDDFT) excitation energies, OSs and spectral assignments for several low-lying valence of $\boldsymbol{P h C l}$.

The lowest UV band $\left(S_{1}\right)$ shows major vibrational structure, and is generally understood to be a $\pi \pi^{*}$ valence state excitation; we concentrate upon the vibrational aspect of the band below. The singlet state manifolds for the $S_{2}$ and $S_{3}$ regions are probably more complex than so far found in the photochemical studies; we record some of the main theoretical results at this point. Of these valence states, we will only describe detailed calculations on the $1^{1} \mathrm{~B}_{2}\left(\mathrm{~S}_{1}\right)$ state.

The AEEs in Table V show that $\pi \pi^{*}$ linear combinations occur. $S_{3}$ is probably simpler than $\mathrm{S}_{2}$ and probably contains both ${ }^{1} \mathrm{~A}_{1}$ and ${ }^{1} \mathrm{~B}_{2}$ components, which are effectively degenerate at the TDDFT and MRD-CI levels of calculations. In the theoretical study of the $\mathrm{S}_{2}$ region at lower energy, dissociation of some states was found at the TDDFT level of theory. Both the ${ }^{1} \mathrm{~B}_{1}\left(3 \mathrm{~b}_{1} 9 \mathrm{a}_{1} *\right)$ and ${ }^{1} \mathrm{~A}_{2}\left(1 \mathrm{a}_{2} 9 \mathrm{a}_{1} *\right)$ states give energy minima at 6.188 and $6.548 \mathrm{eV}$, where the C-Cl bonds are 1.677 and $1.722 \AA$, respectively, which are consistent with bound states. In contrast, the TDDFT module reaches apparent criteria for convergence based on energy gradients, for two other states, ${ }^{1} \mathrm{~B}_{2}\left(6 \mathrm{~b}_{2} 9 \mathrm{a}_{1}{ }^{*}\right) \sigma \sigma^{*}$ and ${ }^{1} \mathrm{~B}_{1}\left(3 \mathrm{~b}_{1} 9 \mathrm{a}_{1}{ }^{*}\right) \pi \sigma^{*}$; but these exhibit $\mathrm{C}$ $\mathrm{Cl}$ bond lengths of $3.327 \AA$ and $2.343 \AA$ respectively, and are merely points on a dissociation path.

The calculated positions of these valence states, which are so prominent in the UV and VUV region, with basis set and with methodology is very variable. Thus, using the aug-ccpVTZ basis set with the TDDFT method, the two bands under $S_{3}$ are well reproduced; however, the lowest band under $S_{1}$ is comparatively high and $S_{2}$ is very low. None of these 
show dramatic geometric changes, although the weak ${ }^{1} \mathrm{~B}_{1}$ component shows more difference. The key issue is the effect of nearby Rydberg states upon the energy of these valence states. This is readily demonstrated by the MRD-CI calculations. The VEEs for the ${ }^{1} \mathrm{~A}_{1}$ major component of $\mathrm{S}_{2}$ is always well reproduced by the MRD-CI study. The ${ }^{1} \mathrm{~A}_{1}$ and ${ }^{1} \mathrm{~B}_{2}$ components of $\mathrm{S}_{3}$ are calculated nearly $0.5 \mathrm{eV}$ high, unless the study includes Rydberg functions, where additional very diffuse components are present in the basis set. The Rydberg functions, especially the d-type functions, have a major impact on the position and intensities of the calculated VEE near $7 \mathrm{eV}$. This is the reason why the present VEEs are closer in energy to experiment than those shown in our previous studies on $\boldsymbol{P h I}$ and $\boldsymbol{P h B r}$, where only valence functions were included.

\section{Detailed aspects of the UV and VUV absorption spectrum of PhCl and its theoretical} interpretation

a. The $1^{1} B_{2}\left(S_{1}\right)$ valence state. An expansion of the UV spectrum in the region of the $S_{1}$ valence state is shown in Fig. 8. The $S_{1}$ state is well known to be due to a $\pi \pi^{*}$ excitation since rotational analysis shows a prolate asymmetric top (B type band) giving an electronic assignment of ${ }^{1} \mathrm{~B}_{2}$ symmetry. ${ }^{68}$ The $\mathrm{S}_{1}$ state band contains sharp vibrational structure (Fig. 8) and has been widely studied experimentally by both $(1+1)^{55,57,69,70}$ and $(2+$ 2) REMPI. ${ }^{71}$ In addition, its lifetime of $749 \mathrm{ps}^{72}$ is sufficiently long that, in contrast to the equivalent state in both $\boldsymbol{P h I}$ and $\boldsymbol{P h B r}$, it has also been studied by fluorescence. ${ }^{73}$

The most recent study of the $S_{1}$ state by Andrejeva et al. ${ }^{55}$ is the most detailed, and used experimental data from $(1+1)$ REMPI spectra of a jet-cooled sample. This technique is very effective in isolating the cold bands in the spectrum and hence enabling accurate frequencies to be determined even when the bands are very weak. However, since the 
spectrum was not normalized to the laser power, the observed intensities are less reliable; for example the intensity of the band origin relative to those around $1000 \mathrm{~cm}^{-1}$ is very different from what is observed in the room-temperature absorption spectrum, which does not suffer from any such power issues. Therefore, in the stick spectrum representation of the experimental data, shown in the middle trace (blue) in Fig. 8(b), the band positions are taken from the REMPI spectrum while the intensities are estimated from the current absorption spectrum.

Andrejeva et al. $^{55}$ assigned their spectrum with the aid of TD-B3LYP/aug-cc-p-TVZ calculations and the calculated frequencies accurately reproduced those observed in the REMPI spectrum. The $\mathrm{S}_{1}$ state frequencies have also been obtained from other $a b$ initio studies. ${ }^{53,73,74}$ We have extended these frequency calculations using the CFOUR package ${ }^{34}$ together with intensity calculations which were not included in the earlier study. ${ }^{55}$ A stick spectrum of the calculated line intensities of the cold bands is shown in the bottom (black) trace of Fig. 8(b). The green trace shows an expansion of part of the low-energy region to highlight the weak bands therein.

Most of our findings confirm the conclusions of the detailed analysis by Andrejeva et $a l .{ }^{55}$ Therefore, we will describe our results only briefly, focussing on those that provide these confirmations and, in one case, offers an alternative assignment to what was reported. First, in the low-wavenumber region, appreciable intensity is calculated for the $20^{2}$ and $14^{2}$ bands, as shown in Fig. 8(b), confirming the proposed assignments. However, we do not calculate any such intensity for the $19^{2}$ band and we suggest that it may gain its intensity by Fermi resonance (FR) with the nearby $10^{1}$ band. Second, FR was invoked for the assignments of the pair of bands $\sim 1050 \mathrm{~cm}^{-1}$ as $10^{1} 11^{1}$ and $7^{1}$, where the former gains its intensity from FR with the latter. We only calculate the presence of one intense band in this region, $7^{1}$, in support of the proposed assignment. 
We have not tried to introduce HT intensity stealing into our current calculations and hence any bands that appear in the experimental spectrum because of this effect will be absent from our simulations. Thus, we did not calculate any intensity for the $30^{1}$ and $29^{1}$ bands which are well-known HT bands and the only such fundamental bands assigned by Andrejeva et al. ${ }^{55}$ In the region around $950 \mathrm{~cm}^{-1}$, we only calculate one intense band, whereas two are observed experimentally and we assign the higher energy of the two, at $965 \mathrm{~cm}^{-1}$, as $28^{1}$. The same assignment was proposed to explain the presence of a very intense peak due to a transition to one quantum of $v_{28}$ in the ground state in the dispersed fluorescence spectrum recorded following excitation of the $965 \mathrm{~cm}^{-1}$ absorption band. ${ }^{73}$ The most obvious absences in our full simulation shown in the lower trace of Fig. 8(a) of HT bands are those induced by $v_{29}$; these are indicated by red asterisks. Those bands induced by $v_{28}$, indicated by blue asterisks, are less noticeable as there are always other strong bands in the same energy region.

The calculated sequence band structure is shown in the inset of Fig. 8(a). This is in very good agreement with both the experimental spectrum and in the reported assignments ${ }^{75}$ and is a further indication of the accuracy of the calculated geometry.

b. Rydberg states The UV and VUV absorption spectrum of $\boldsymbol{P h C l}$ at room temperature is shown in Fig. 1(b). In order to identify a significant number of Rydberg states, which are intrinsically weak but which exhibit sharp vibrational structure, as observed in their PES spectra (Figs. 4 and 6), we need a procedure to remove broad features of whatever origin. A method previously used to simplify the VUV spectrum of $\boldsymbol{P h I}$ and $\boldsymbol{P h} \boldsymbol{B r} \boldsymbol{r}^{2,3}$ was applied to that for $\boldsymbol{P h} \boldsymbol{C l}$. Briefly, in order to help identify features in the VUV spectrum as Rydberg states, an underlying structure made up of several very broad Gaussian bands was 'subtracted' from the original spectrum. Whilst we do not exclude valence states exhibiting fine structure 
in the energy region above $6 \mathrm{eV}\left(48393 \mathrm{~cm}^{-1}\right)$, only the sharp Rydberg states are of particular interest.

The resultant fine structure allowed many Rydberg states to be identified by comparison of the local vibrational structure with individual PES band envelopes; the resulting transition energies of the electronic origins of the states are shown in Tables VI, VII and VIII. Also shown in these Tables are the effective quantum numbers, $n^{*}$, values of the origins, calculated using the conventional Rydberg formula and the appropriate IE (see Table I), which are used as an aid in the assignment of the $n l$ values of the states. Although the most intense peak in the PES of the $\mathrm{A}^{2} \mathrm{~A}_{2}$ ionic state $\left(78327 \mathrm{~cm}^{-1}\right)$ lies in the region over which VUV spectra was recorded, some Rydberg series converging on this limit, are likely to be present in Fig. 1b, but are not conclusively identified and will not be discussed here.

The most intense Rydberg bands are in the series converging on the $\mathrm{B}^{2} \mathrm{~B}_{2}$ state of the ion as expected from the PES spectrum where most of the OS of this state is concentrated in a narrow origin band. The most intense Rydberg band in the absorption spectrum of $\boldsymbol{P h} \boldsymbol{C l}$ is the origin of the [3] $3 \mathrm{~s}$ state at $68611 \mathrm{~cm}^{-1}$. This $n$ s-series can be traced up to $n=5$. In addition, two $n$ p- and two $n$ d-series have been identified as shown in Fig. 9; the equivalent series converging on $\mathrm{IE}_{3}$ were also identified in the spectrum of $\boldsymbol{P h} \boldsymbol{B r}$. In addition, several [4] Rydberg states are

The fingerprint of both the $\mathrm{B}^{2} \mathrm{~B}_{2}$ and $\mathrm{C}^{2} \mathrm{~B}_{1}$ states of the ion, and therefore also Rydberg series converging on them, is a dominant origin making them relatively easy to identify. However, the $\mathrm{X}^{2} \mathrm{~B}_{1}$ band in the PES contains at least five bands of appreciable intensity that, to a first approximation, are equally spaced (our calculations show that they are not due to one progression of a single vibration). For example, the vibrational structure observed in the PES of the $X^{2} B_{1}$ state, the VUV absorption spectrum of the $[1] 3 d_{3}$ state and the $(2+1)$ REMPI of the $[1] 4 \mathrm{~d}_{1}$ state is shown in Figs. $10(\mathrm{a}),(\mathrm{b})$ and (c), respectively. This extended vibrational 
structure makes identification of electronic origins more uncertain and therefore, we began this part of the analysis by transferring the binding energies of the Rydberg states converging on this state of the ion observed in the VUV spectrum of $\boldsymbol{P h B r}$ to the $\boldsymbol{P h} \boldsymbol{C l}$ spectrum. A set of assignments was derived that accounts for all the observed bands and this is illustrated in Figs. 11, 12 and 13. A few origins are obscured by more intense bands of [3] Rydberg states and these are highlighted in Table VI.

\section{E. $(2+1)$ REMPI spectrum}

Various factors enabled us to obtain more extensive and detailed information about the Rydberg states from $(2+1)$ REMPI spectra of jet-cooled $\boldsymbol{P h} \boldsymbol{C l}$. First, the propensities for 1and 2-photon transitions are different and hence more states are observable, e.g. the two $4 \mathrm{f}$ states observed in the 1-photon spectrum are not seen in the 2-photon spectrum and vice versa for the $4 d_{1}$ state as shown in Fig. 12. The $(2+1)$ REMPI spectrum is dominated by two [1]nd Rydberg series, one of which has $A_{1}$ symmetry, that merge for $n>11$ and can be identified up to $n=16$. The transition energies of the electronic origins are tabulated in Table VI, together with their quantum defects calculated using $\mathrm{IE}_{1}$ of $73172 \mathrm{~cm}^{-1}$ measured by $\mathrm{Ng} .{ }^{13}$ To within the limits of the resolution of the VUV spectrum, it appears that one series, $n \mathrm{~d}_{2}$, is common to both 1- and 2-photon spectra; only the 2-photon transition energies for this series are presented. No $n \mathrm{~s}^{-}, n \mathrm{p}$ or $n \mathrm{f}$ states are identified in the $(2+1)$ REMPI spectrum.

Second, the resolution in the spectra is much greater since a narrow bandwidth $(\sim 0.2$

$\mathrm{cm}^{-1}$ ) laser was used and the sample was rotationally and vibrationally cooled in the molecular beam. Thus the natural band widths, e.g. $\sim 15 \mathrm{~cm}^{-1}$ for the $12 \mathrm{~d}$ states, are observed experimentally. It can be seen that the widths of the peaks in the REMPI spectrum decrease as the energy increases, e.g. $\sim 40 \mathrm{~cm}^{-1}$ for the $4 \mathrm{~d}$ states to $\sim 15 \mathrm{~cm}^{-1}$ for the $10 \mathrm{~d}$ states, which we 
attribute to a decrease in the degree of interaction with underlying valence states resulting in an increase in the Rydberg state lifetime. Experimentally, this decrease in coupling means that the Rydberg peaks become more observable for two reasons. First, the peaks are more discernable above the background signal. Second, the increase in lifetime means that the ionization efficiency increases, as this has to compete with radiative decay. The valence states themselves clearly have very low ionization efficiencies as none are observed in the REMPI spectrum either because of very short lifetimes and/or small FCFs with the ground state of the ion. A combination of these two factors mean that in the $[1] 5 \mathrm{~d} /[3] 3 \mathrm{~s}, 3 \mathrm{p}$ region the former is seen uniquely in the REMPI spectrum while the latter are only observed in the VUV absorption spectrum as shown in Fig. 13.

Third, the symmetry of some of the states can be determined by REMPI polarization data, e.g. a band whose intensity is smaller in the spectrum recorded with circularly polarized (CP) light than it is in one recorded with linearly polarized (LP) light, a $\boldsymbol{P}_{-}$band, must be due to a totally symmetric transition. Thus, an electronic origin accessed by a $\mathcal{P}_{-}$cold band must be that of a totally symmetric state, i.e. $\mathrm{A}_{1}$ in $\mathrm{C}_{2 \mathrm{v}}$ symmetry, whereas all other states accessed by $\mathcal{P}_{+}$cold bands must have $A_{2}, B_{1}$ or $B_{2}$ symmetry; an example is shown in Fig. 14 where the $n \mathrm{~d}_{2}$ series must have $\mathrm{A}_{1}$ symmetry.

\section{F. Rydberg state intensities in various spectra}

$\boldsymbol{P h C l}$ provides a good example of how the results of studies that use different excitation pathways can be combined to identify the energies, and in some cases the symmetries, of a wider range of Rydberg states than is possible from a single technique. Specifically, we consider the $4 \mathrm{p}$ and $4 \mathrm{~d}$ Rydberg states as studied by 1-photon (VUV) 
absorption (Figs. 11 and 12(a)), non-resonant 2-photon $((2+1)$ REMPI) (Fig. 13(b)) and resonant 2-photon, (DR-MPI-PES) via the $S_{1}$ state). ${ }^{27}$

It is probable that all five $4 \mathrm{~d}$ Rydberg state origins are observed in the three spectra combined. The $\mathrm{A}_{1}$ component $\left(n \mathrm{~d}_{2}\right)$ is the $(2+1)$ REMPI band at $66004 \mathrm{~cm}^{-1}$ as shown by the polarization behaviour. The $\mathrm{A}_{2}$ component $\left(n \mathrm{~d}_{4}\right)$ is probably the DR-MPI-PES band at 66390 $\mathrm{cm}^{-1}$ as $\mathrm{A}_{2}$ bands are the most intense in this energy region when the $S_{1}$ state origin is used as the intermediate level. ${ }^{27}$ The $3 \mathrm{~d} \mathrm{~B}_{2}$ component was assigned ${ }^{27}$ to a very broad band at 60300 $\mathrm{cm}^{-1}$ in the DR-MPI-PES and we propose that the $4 \mathrm{~d}$ equivalent $\left(n \mathrm{~d}_{1}\right)$ is the unassigned broad band $\sim 66080 \mathrm{~cm}^{-1}$ in the same spectrum. Finally, we assign the two $\mathrm{B}_{1}$ components $\left(n \mathrm{~d}_{3}\right)$ and $\left(n \mathrm{~d}_{5}\right)$ to the 1-photon bands at 66335 and $66551 \mathrm{~cm}^{-1}$, respectively.

To within the quoted accuracy of the measurements in the various spectra, only the band at $64690 \mathrm{~cm}^{-1}$ in the DR-MPI-PES and $64683 \mathrm{~cm}^{-1}$ in the 1-photon spectrum could possibly be assigned to the same state. However, the DR-MPI-PES band was shown unambiguously ${ }^{27}$ to be due to an $\mathrm{A}_{2}$ electronic origin and a 1-photon transition to a state of this symmetry is forbidden and hence the peaks in the two spectra must be due to different, overlapping states with the $n^{*}$ value of 3.60 pointing to them being $4 p$ Rydberg states. The third $4 \mathrm{p}$ state is assigned to the 1-photon band at $64412 \mathrm{~cm}^{-1}$ and the bands in the 1-photon spectrum must be due to states with $\mathrm{A}_{1}$ and $\mathrm{B}_{1}$ symmetry.

In general, it appears that there are very few states that are observed with large intensities in more than one of the three different spectra and that symmetry restrictions alone cannot account for this. In particular, no $n p$ Rydberg states are observed in the $(2+1)$ REMPI spectrum even though transitions to all three are allowed whereas the $4 p A_{2}$ band is very intense in the DR-MPI-PES. Thus, the absence of the same band in the $(2+1)$ REMPI spectrum is not due to a prohibitively low ionization efficiency but must be due to a very small 2-photon transition cross section. In a subsequent Paper $^{60}$ we will show that there is the 
same absence of $n$ p Rydberg states in the non-resonant 2-photon spectrum of $\boldsymbol{P h F}$. In benzene itself, such transitions are symmetry forbidden and we propose that this shows that the light halogen atoms, $\mathrm{F}$ and $\mathrm{Cl}$ have little effect on the 2-photon transition cross sections.

In our subsequent Paper $^{60}$ on $\boldsymbol{P h} \boldsymbol{F}$ we derive additional information from $(3+1)$ REMPI spectra. While we could readily obtain 3-photon spectra of $\boldsymbol{P h} \boldsymbol{F}$ by collecting $\mathrm{C}^{+}$and $\mathrm{C}_{2}{ }^{+}$in the region 69000 to $72000 \mathrm{~cm}^{-1}$, only very broad features were observed and we have not observed any structure that can be assigned to excitation of Rydberg states. We observed a large increase in signal $\sim 69000 \mathrm{~cm}^{-1}$ which is probably due to the onset of absorption into the $\mathrm{S}_{2}$ state at the 2-photon level. At the low energy side of this onset, there is 2-photon excitation of the high-energy pseudo-continuum of the $S_{1}$ state and this may lead to dissociation, thus preventing 3-photon excitation of Rydberg states.

\section{CONCLUSIONS}

The availability of a new VUV absorption spectrum of $\boldsymbol{P h} \boldsymbol{C l}$, which had not previously been analyzed for the presence of Rydberg states, necessitated a new photoelectron study. AIEs determined using both MCSCF and EOM-CCSD procedures, determined the energy order as: $\mathrm{X}^{2} \mathrm{~B}_{1}<\mathrm{A}^{2} \mathrm{~A}_{2}<\mathrm{B}^{2} \mathrm{~B}_{2}<\mathrm{C}^{2} \mathrm{~B}_{1}$, although the two highest states were very close in energy.

Both the VUV and PES studies include vibrational analysis by theoretical means which includes both hot and cold band analyses. Our calculations show that hot, sequence band intensities contribute significantly to the observed PES profiles; this is especially convincing for the asymmetric band shapes in the $\mathrm{X}^{2} \mathrm{~B}_{1}$ state. The calculated FC vibrational spectral envelope for the $\mathrm{X}^{2} \mathrm{~B}_{1}$ including both hot and cold band contributions, reproduces the observed peak positions and intensities with high accuracy. The vibrational analysis shows 
that many PES individual peaks are summations of several vibrations, and hence more complex than previously thought; only a PES study at much higher resolution will confirm these predictions. Only totally symmetric vibrations give rise to bands with appreciable intensity in the FC simulation, and these include all modes except $\mathrm{C}-\mathrm{H}$ stretches.

As with our previous $\boldsymbol{P h B r}$ studies, ${ }^{3}$ our software only allows study of individual electronic states, and we cannot study the build-up of intensity in the energy region between the $\mathrm{X}^{2} \mathrm{~B}_{1}$ and $\mathrm{A}^{2} \mathrm{~A}_{2}$ states; this is attributed to vibronic coupling between the two states. Specifically, any bands that gain their intensity via this mechanism will not appear in our simulations and hence can be distinguished from those that gain their intensity purely from FC overlap. From a comparison of our simulations with, particularly, the MATI spectra, we propose that two $b_{2}$ vibrational modes, $v_{23}$ and $v_{24}$, are most effective in inducing this vibronic coupling. Only two broad peaks are observed in the $\mathrm{A}^{2} \mathrm{~A}_{2}$ state band and we propose that the first, more intense of the two is due to a maximum in the superimposed vibronically induced $\mathrm{X}^{2} \mathrm{~B}_{1}$ vibrational bands and electronically allowed $\mathrm{A}^{2} \mathrm{~A}_{2}$ bands and not to the adiabatic IE which lies to lower energy.

Both the energy ordering and the calculated FC vibrational spectral envelopes for the $\mathrm{B}$ and $\mathrm{C}$ bands strongly suggest the assignments to ${ }^{2} \mathrm{~B}_{2}$ and ${ }^{2} \mathrm{~B}_{1}$, respectively; this is in agreement with most previous studies but is in conflict with previous PIRI studies. ${ }^{64} \mathrm{We}$ attribute the discrepancies in the simulations of these two bands and in the need for an increased bandwidth for the upper state to coupling between the two.

In general, our procedures for calculating FC profiles are shown to be excellent for the uncoupled regions of ionic states of $\boldsymbol{P h} \boldsymbol{X}$ molecules. While they are not so effective in simulating coupled regions, they can give some, less detailed, information on the vibrational structure and also identify regions where coupling occurs. 
The interpretation of the experimental UV and VUV spectrum of $\boldsymbol{P h} \boldsymbol{C l}$ in the $4-11 \mathrm{eV}$ range is by far the most detailed yet reported. The vibrational structure in the spectra has been analyzed using a combination of adiabatic TDDFT and vertical (MRD-CI) sets of calculations; the band origins between theory and experiment require only small adjustments of the energy scale (generally less than $0.4 \mathrm{eV}$ (AEE) and $0.25 \mathrm{eV}$ (VEE)) to superimpose the FC vibrational structure from the calculations onto the main experimental bands. However, very diffuse functions are essential in the basis set for acceptable VEEs especially for the ${ }^{1} \mathrm{~A}_{1}$ manifold; this is not to say that the states in question under the $\mathrm{S}_{3}$ band have high Rydberg state character; it is that in any symmetry manifold, each state in a multi-reference multi-root calculation is determined in the field of the other states of same symmetry. In short, adjacent electronic states compete for the energy range, and this can lead to states, including valence states, being 'forced' to lower or higher energies, by interstate competition.

In agreement with previous work, the $S_{1}$ band $\left(4.6 \mathrm{eV}, 37000 \mathrm{~cm}^{-1}\right)$ has been shown to be due to the $1^{1} \mathrm{~B}_{2}$ state. As described above for the $\mathrm{X}^{2} \mathrm{~B}_{1}$ ionic state, only some of the experimental structure is explicable in terms of the FC analyses presented. The remainder is confirmed as being due to HT intensity stealing via the $\mathrm{b}_{2}$ vibrations, $v_{28}, v_{29}$ and $v_{30}$; a phenomenon well known in this state ${ }^{55,57,73}$ and its equivalent in $\boldsymbol{P h F} .^{76}$

We have made extensive use of FC vibrational profiles observed in the PES to identify the main vibrational progressions occurring in the VUV absorption spectra. A considerable number of Rydberg states were identified at higher energy in the spectrum and were categorized, largely, by comparison with the VUV spectrum of $\boldsymbol{P h I}$ and $\boldsymbol{P h} \boldsymbol{B r}{ }^{2,3}$ The use of the vibrational profiles from the PES spectrum is critical to these identifications, owing to the density of states apparent in the VUV spectrum, and in that respect, the TDDFT method proved valuable. Using this approach, a considerable number of Rydberg series converging on 
the $\mathrm{X}^{2} \mathrm{~B}_{1}$ and $\mathrm{B}^{2} \mathrm{~B}_{2}$ states have been identified, a few converging on the $\mathrm{C}^{2} \mathrm{~B}_{1}$ state have been tentatively assigned, but none converging on the $\mathrm{A}^{2} \mathrm{~A}_{2}$ state have been observed.

A large number of other Rydberg states converging on the $\mathrm{X}^{2} \mathrm{~B}_{1}$ ionic state have been observed in the $(2+1)$ REMPI spectrum and the symmetries of some of these have been directly identified from polarization data. This analysis has led to the indirect assignment of the symmetries of several other Rydberg states observed in both the VUV absorption and (1+ $\left.1^{\prime}\right)$ REMPI spectra. ${ }^{27}$ The combination of the results of several experimental techniques described here provides a good illustration of how the final conclusions are greater than the sum of their parts and we will extend this approach even further in a subsequent Paper on $\boldsymbol{P h F}^{60}$

\section{Acknowledgements}

We thank: (a) the European Community's Seventh Framework Programme (FP7/20072013) CALIPSO under grant agreement $n^{\circ} 312284$ for supporting the research leading to these results; (b) the Elettra Synchrotron facility for a Grant of beamtime; (c) the ASTRID2 facility for grants to carry out the parallel VUV absorption study; (d) the NSCCS super-computing facility of the UK for support; (e) the Italian MIUR (under the projects PON01-01078/8, PRIN 2011 DEMOCRITOS and PRIN 2010ERFKXL 006); (f) Prof. Vincenzo Barone and Dr. Julien Bloino for helpful discussions; (g) C. Puglia (Uppsala University, Sweden) and the Carl Tygger Foundation for making available the VG-Scienta SES-200 photoelectron analyser at the Gas Phase beamline, Elettra, Italy. 


\section{References.}

${ }^{1}$ M. H. Palmer, T. Ridley, S. Vrønning Hoffmann, N. C. Jones, M. Coreno, M. de Simone, C. Grazioli, M. Biczysko, and A. Baiardi, J. Chem. Phys. 142, 134301 (2015).

${ }^{2}$ M. H. Palmer, T. Ridley, S. Vrønning Hoffmann, N. C. Jones, M. Coreno, M. de Simone, C. Grazioli, M. Biczysko, A. Baiardi, and P. M. Limão-Vieira, J. Chem. Phys. 142, 134302 (2015).

${ }^{3}$ M. H. Palmer, T. Ridley, S. Vrønning Hoffmann, N. C. Jones, M. Coreno, M. de Simone, C. Grazioli, T. Zhang, M. Biczysko, A. Baiardi and K. Peterson, J. Chem. Phys. 143, 164303 (2015).

${ }^{4}$ M. S. Child, Theory of Molecular Rydberg States (Cambridge University Press, Cambridge, 2011).

${ }^{5}$ T. F. Gallagher, Rydberg Atoms (Cambridge University Press, Cambridge, 1994).

${ }^{6}$ R. S. Mulliken, J. Am. Chem. Soc. 86, 3183 (1964).

${ }^{7}$ R. S. Mulliken, J. Am. Chem. Soc. 88, 1849 (1966).

${ }^{8}$ M. H. Palmer, S. Vronning Hoffmann, N. C. Jones, A. R. Head, and D. L. Lichtenberger, J. Chem. Phys. 134, 084309 (2011).

${ }^{9}$ M. H. Palmer, P. J. Camp, S. Vronning Hoffmann, N. C. Jones, A. R. Head, and D. L. Lichtenberger, J. Chem. Phys. 136, 094310 (2012).

${ }^{10}$ M. H. Palmer, S. Vrønning Hoffmann, N. C. Jones, E. R. Smith, and D. L. Lichtenberger, J. Chem. Phys. 138, 214317 (2013).

${ }^{11}$ C. H. Kwon, H. L. Kim, and M. S. Kim, J. Chem. Phys. 116, 10361 (2002).

12 A. W. Potts, D. Edvardsson, L. Karlsson, D. M. P. Holland, M. A. MacDonald, M. A. Hayes, R. Maripuu, K. Siegbahn, and W. von Niesson, Chem. Phys. 254, 385 (2000). 
${ }^{13}$ C-H. Ng, Ann. Rev. Phys. Chem. 65, 197 (2014).

${ }^{14}$ M. B. Robin, Higher excited states of polyatomic molecules (Academic Press, New York, 1974), Vol. 2.

${ }^{15}$ S-F. Chen, F-Y. Liu, and Y-J. Liu, J. Chem. Phys. 131, 124304 (2009).

${ }^{16}$ T. Ichimura, Y. Mori, H. Shinohara, and N. Nishi, Chem. Phys. 189, 117 (1994).

${ }^{17}$ G-J. Wang, R-S. Zhu, H. Zhang, K-L. Han, G-Z. He, and N-Q. Lou, Chem. Phys. Lett. 288, 429 (1998).

${ }^{18}$ M. Kadi, J. Davidsson, A. N. Tarnovsky, M. Rasmusson, and E. Akesson, Chem. Phys. Lett. 350, 93 (2001).

${ }^{19}$ T. Ichimura, Y. Mori, H. Shinohara, and N. Nishi, Chem. Phys. 122, 51 (1985).

${ }^{20}$ A. Freedman, S. C. Yang, M. Kawasaki, and R. Bersohn, J. Chem. Phys. 72, 1028 (1980).

${ }^{21}$ L. Sobolewski and W. Domcke, Chem. Phys. 259, 181 (2000).

${ }^{22}$ Y-J. Liu, P. Persson, and S. Lunell, J. Chem. Phys. 121, 11000 (2004).

${ }^{23}$ W. C. Price and A. D. Walsh, Proc. Royal Soc. London, Series A 191, 22 (1947).

${ }^{24}$ M. B. Robin, Higher excited states of polyatomic molecules (Academic Press, New York, 1985), Vol. 3.

${ }^{25}$ K. Kimura and S. Nagakura, Spectrochim. Acta 17, 166 (1961).

${ }^{26}$ K. Kimura and S. Nagakura, Mol. Phys. 9, 117 (1965).

${ }^{27}$ P. Asselin, F. Piuzzi, J. Le Calvé, M. Mons, and I. Dimicoli, Chem. Phys. 181, 271 (1994).

${ }^{28}$ K. C. Prince, R. R. Blyth, R. Delaunay, M. Zitnik, J. Krempasky, J. Slezak, R. Camilloni, L. Avaldi, M. Coreno, G. Stefani, C. Furlani, M. de Simone, and M. Stranges, J. Synchrotron Radiat. 5, 565 (1998).

${ }^{29}$ See supplementary material at XXXX. Wide scan PES, variation in the ground, ionic and electronically excited state structures, FC ionic state calculated frequencies and intensities, further details of the MRD-CI vertical excitation to singlet states. 
${ }^{30}$ R. Bauernschmitt and R. Ahlrichs, Chem. Phys. Lett. 256, 454 (1996).

${ }^{31}$ M. E. Casida, C. Jamorski, K. C. Casida, and D. R. Salahub, J. Chem. Phys. 108, 4439 (1998).

${ }^{32}$ R. E. Stratmann, G. E. Scuseria, and M. J. Frisch, J. Chem. Phys., 109, 8218 (1998).

${ }^{33}$ M. J. Frisch, G. W. Trucks, H. B. Schlegel, G. E. Scuseria, M. A. Robb, J. R. Cheeseman, G. Scalmani, V. Barone, B. Mennucci, G. A. Petersson, H. Nakatsuji, M. Caricato, X. Li, H. P. Hratchian,A. F. Izmaylov, J. Bloino, G. Zheng, J. L. Sonnenberg, M. Hada, M. Ehara, K. Toyota, R. Fukuda, J. Hasegawa, M. Ishida, T. Nakajima, Y. Honda, O. Kitao, H. Nakai, T. Vreven, J. A. Montgomery, Jr., J. E. Peralta, F. Ogliaro, M. Bearpark, J. J. Heyd, E. Brothers, K. N. Kudin, V. N. Staroverov, T. Keith, R. Kobayashi, J. Normand, K. Raghavachari, A. Rendell, J. C. Burant, S. S. Iyengar, J. Tomasi, M. Cossi, N. Rega, J. M. Millam, M. Klene, J. E. Knox, J. B. Cross, V. Bakken, C. Adamo, J. Jaramillo, R. Gomperts, R. E. Stratmann, O. Yazyev, A. J. Austin, R. Cammi, C. Pomelli, J. W. Ochterski, R. L. Martin, K. Morokuma, V. G. Zakrzewski, G. A. Voth, P. Salvador, J. J. Dannenberg, S. Dapprich, A. D. Daniels, O. Farkas, J. B. Foresman, J. V. Ortiz, J. Cioslowski, and D. J. Fox, Gaussian 09, Revision D.01, Gaussian, Inc., Wallingford CT, 2013.

${ }^{34}$ CFOUR, Coupled-Cluster techniques for Computational Chemistry, a quantum-chemical program package by J. F. Stanton, J. Gauss, M. E. Harding, P. G. Szalay with contributions from A. A. Auer, R. J. Bartlett, U. Benedikt, C. Berger, D. E. Bernholdt, Y. J. Bomble, L. Cheng, O. Christiansen, M. Heckert, O. Heun, C. Huber, T.-C. Jagau, D. Jonsson, J. Jusélius, K. Klein, W. J. Lauderdale, F. Lipparini, D. A. Matthews, T. Metzroth, L. A. Mück, D. P. O'Neill, D. R. Price, E. Prochnow, C. Puzzarini, K. Ruud, F. Schiffmann, W. Schwalbach, C. Simmons, S. Stopkowicz, A. Tajti, J. Vázquez, F. Wang, J. D. Watts and the integral packages MOLECULE (J. Almlöf and P. R. Taylor), PROPS (P. R. Taylor), ABACUS (T. 
Helgaker, H. J. A. Jensen, P. Jørgensen, and J. Olsen), and ECP routines by A. V. Mitin and

C. van Wüllen. For the current version, see http://www.cfour.de.

${ }^{35}$ J. F. Stanton and J. Gauss, J. Chem. Phys. 101, 8938 (1994).

${ }^{36}$ H.-J. Werner, P. J. Knowles, F. R. Manby, M. Schütz, P. Celani, T. Korona, R. Lindh, A. Mitrushenkov, G. Rauhut, K. R. Shamasundar, T. B. Adler, R. D. Amos, A. Bernhardsson, A. Berning, D. L. Cooper, M. J. O. Deegan, A. J. Dobbyn, F. Eckert, E. Goll, C. Hampel, A. Hesselmann, G. Hetzer, T. Hrenar, G. Jansen, C. Köppl, Y. Liu, A. W. Lloyd, R. A. Mata, A. J. May, S. J. McNicholas, W. Meyer, M. E. Mura, A. Nicklaß, D. P. O'Neill, P. Palmieri, K. Pflüger, R. Pitzer, M. Reiher, T. Shiozaki, H. Stoll, A. J. Stone, R. Tarroni, T. Thorsteinsson, M. Wang, and A. Wolf, MOLPRO Version 2012.1 a package of ab initio programs, 2012, see http://www.molpro.net.

37 V. Barone, J. Bloino, and M. Biczysko, Vibrationally-resolved electronic spectra in GAUSSIAN 09 Revision A.02 (2009) http://dreamslab.sns.it, accessed July01, 2015.

${ }^{38}$ J. Bloino, M. Biczysko, O. Crescenzi, and V. Barone, J. Chem. Phys. 128, 244105 (2008).

${ }^{39}$ V. Barone, J. Bloino, M. Biczysko, and F. Santoro, J. Chem. Theory Comput. 5, 540 (2009).

${ }^{40}$ J. Bloino, M. Biczysko, F. Santoro, and V. Barone, J. Chem. Theory Comput. 6, 1256 (2010).

41 R. J. Buenker, in Current Aspects of Quantum Chemistry, edited by R. Carbo (Elsevier, New York, 1982), p. 17.

${ }^{42}$ R. J. Buenker and R. A. Phillips, J. Mol. Struct.: THEOCHEM 24, 291 (1985).

${ }^{43}$ R. J. Buenker and S. Krebs, in Recent Advances in Multireference Methods, edited by K. Hirao (World Scientific, Singapore, 1999), p. 1.

${ }^{44}$ M. F. Guest, I. J. Bush, H. J. J. Van Dam, P. Sherwood, J. M. H. Thomas, J. H. Van Lenthe, R. W. A. Havenith, and J. Kendrick, Mol. Phys. 103, 719 (2005). 
${ }^{45}$ T. H. Dunning, Jr., J. Chem. Phys. 90, 1007 (1989).

${ }^{46}$ R. A. Kendall, T. H. Dunning, Jr., and R. J. Harrison, J. Chem. Phys. 96, 6796 (1992).

${ }^{47}$ D. E. Woon and T. H. Dunning, Jr., J. Chem. Phys. 98, 1358 (1993).

${ }^{48}$ A. D. McLean and G. S. Chandler, J. Chem. Phys. 72, 5639 (1980).

${ }^{49}$ R. C. Binning and L. A. Curtiss, J. Comput. Chem. 11, 1206 (1990).

${ }^{50}$ M. Piccardo, J. Bloino, and V. Barone, Int. J. Quant. Chem. 115, 948 (2015).

${ }^{51}$ V. Barone, M. Biczysko, and J. Bloino, Phys. Chem. Chem. Phys. 16, 1759 (2014).

${ }^{52}$ I. Carnimeo, C. Puzzarini, N. Tasinato, P. Stoppa, A. P. Charmet, M. Biczysko, C.

Cappelli, and V. Barone, J. Chem. Phys. 139, 074310 (2013).

${ }^{53}$ W-Z Li and M-B Huang, Chem. Phys. Lett. 405, 1 (2005).

${ }^{54}$ A. M. Gardner and T. G. Wright, J. Chem. Phys. 135, 114305 (2011).

${ }^{55}$ A. Andrejeva, W. D. Tuttle, J. P. Harris, and T. G. Wright, J. Chem. Phys. 134, 084309 (2011).

${ }^{56}$ P. Asselin, A. Gouzerh, F. Piuzzi, and I. Dimicoli, Chem. Phys. 175, 387 (1993).

${ }^{57}$ T. Wright, S. I. Panov, and T. A. Miller, J. Chem. Phys. 102, 4793 (1995).

${ }^{58}$ I. Bâldea, J. Franz, P. G. Szalay, and H. Köppel, Chem. Phys. 329, 65 (2006).

${ }^{59}$ E. Gindensperger, I. Bâldea, P. G. Szalay, and H. Köppel, Chem. Phys. 338, 207 (2007).

${ }^{60}$ M. H. Palmer, T. Ridley, S. Vrønning Hoffmann, N. C. Jones, M. Coreno, M. de Simone, C. Grazioli, M. Biczysko, A. Baiardi, and K. Peterson, "Combined theoretical and experimental study of the valence, Rydberg and ionic states of fluorobenzene', (unpublished).

${ }^{61}$ Y. Y. Youn, C. H. Kwon, J. C. Choe, and M. S. Kim, J. Chem. Phys. 117, 2538 (2002).

${ }^{62}$ S. Fujisawa, K. Ohno, S. Masuda, and Y.Harada, J. Am. Chem. Soc., 108, 6505 (1986). 
${ }^{63}$ S-Y. Yu and M-B. Huang, Chem. Phys, 328, 291 (2006).

${ }^{64}$ R. Anand, J. D. Hofstein, J. E. Le Claire, P. M. Johnson, and C. Cossart-Magos, J. Phys. Chem. A 103, 8927 (1999).

${ }^{65}$ P. M. Johnson, R. Anand, J. D. Hofstein, and J. E. Le Claire, J. Elect. Spectrosc. Relat. Phenom. 108, 177 (2000).

${ }^{66}$ X. Ripoche, I. Dimicoli, J. Le Calvé, F. Piuzzi, and R. Botter, Chem. Phys. 124, 305 (1988).

${ }^{67}$ K. Walter, U. Boesl, and E. W. Schlag, Chem. Phys. Lett. 162, 261 (1989).

${ }^{68}$ T. Cvitaš and J. M. Hollas, Molec. Phys., 18, 101 (1970).

${ }^{69}$ K. Walter, K. Scherm, and U. Boesl, J. Phys. Chem. 95, 1188 (1991).

${ }^{70}$ S. L. Anderson, D. M. Rider, and R. N. Zare, Chem. Phys. Lett. 93, 11 (1982).

${ }^{71}$ J. Murakami, K. Kaya, and M. Ito, J. Chem. Phys. 72, 3263 (1980).

${ }^{72}$ Y-Z. Liu, C-C. Qin, S. Zhang, Y-M. Wang, and B. Zhang, Wuli Huaxue Xuebo 26, 965 (2011).

${ }^{73}$ P. Imhoff and K. Kleinermanns, Chem. Phys. 270, 227 (2000).

${ }^{74}$ P. Imhoff, D. Krügler, R. Brause, and K. Kleinermanns, J. Chem. Phys. 121, 2598 (2004).

${ }^{75}$ Y. S. Jain and H. D. Bist, J. Mol. Spectrosc. 47, 126 (1973).

${ }^{76}$ I. Pugliesi, N. M. Tonge, and M. C. R. Cockett, J. Chem. Phys. 129, 104303 (2008). 


\section{Figure Captions.}

Figure 1. Low-energy region of the PES, (a), and the VUV absorption spectra, (b), of $\boldsymbol{P h C l}$. The [3]3s Rydberg state peak, where [3] indicates a Rydberg state with a third lowest, $\mathrm{B}^{2} \mathrm{~B}_{2}$, ionic core, is the most intense Rydberg peak as is the $\mathrm{B}^{2} \mathrm{~B}_{2}$ origin in the PES spectrum.

Figure 2. The experimental UV and VUV absorption spectrum of $\boldsymbol{P h} \boldsymbol{C l}$, (black) with inset the lowest ${ }^{1} \mathrm{~B}_{2}$ state with intensity increased by a factor of 150 . Also superimposed are the all-electron MRD-CI energies and oscillator strengths for calculated valence states (red), where the basis set includes Rydberg functions. Positions of ${ }^{1} \mathrm{~A}_{2}$ states are included by use of an arbitrary $f(r)=10^{-4}$. A logarithmic plot of the calculated oscillator strengths, which emphasizes the variation in $\mathrm{f}(\mathrm{r})$ with energy is shown in the supplementary material. ${ }^{29}$

Figure 3. Constant density contour diagrams of $\boldsymbol{P h C l}$. The four highest occupied MOs, (a); when ionized the sequence is maintained as $3 b_{1}{ }^{-1}<1 a_{2}{ }^{-1}<6 b_{2}{ }^{-1}<2 b_{1}{ }^{-1}$. The four lowest valence unoccupied (virtual) MOs, (b); in the low $\pi \sigma^{*}$ excited singlet states, the $8 \mathrm{a}_{1} *$ MO is occupied.

Figure 4. Stick spectrum of the most intense lines for the non-sequence bands of the $\mathrm{X}^{2} \mathrm{~B}_{1}$ PES band of $\boldsymbol{P h} \boldsymbol{C l}$, (a), in which the color-coded asterisks indicate progressions of $\boldsymbol{v}_{11}$ built on the electronic origin and the labelled vibrational fundamentals, e.g. peaks labelled in green show $7^{1} 11^{\mathrm{n}}$ excited states. The experimental spectrum (black trace) is shown in (b) together with a simulation (red trace) obtained by convoluting the full stick spectrum with a bandwidth of $150 \mathrm{~cm}^{-1}$. The simulation is scaled such that the intensity of the origin peak matches that of the experimental spectrum. The 1-photon MATI spectrum (blue trace), ${ }^{11}$ with intensity scaled to match the PES origin and offset for display purposes, is also shown in (b). The peaks in the MATI spectrum labelled with black and red '+'s are $23^{1} 11^{n}$ and $24^{1} 11^{n}$ bands, respectively. A stick spectrum of the calculated wavenumber shifts of the most intense 
sequence bands associated with the $\mathrm{X}^{2} \mathrm{~B}_{1}$ electronic origin peak (lower black trace), inset of (a). The first, $n=1$, member of a $v_{\mathrm{N}}$ sequence band series, $\mathrm{N}^{1}{ }_{1}$ is labelled; $n>1$ members are denoted by color-coded asterisks. A convolution of this spectrum with a bandwidth of $150 \mathrm{~cm}^{-}$ 1 is shown in the red trace and the upper black trace shows the experimental band appropriately offset in energy.

Figure 5. Stick spectrum of the calculated line intensities for the $\mathrm{A}^{2} \mathrm{~A}_{2}$, band of $\boldsymbol{P h} \boldsymbol{C l}$, (a). The experimental spectrum is shown in the black trace in (b) together with a simulation obtained by convoluting the stick spectrum with a bandwidth of $600 \mathrm{~cm}^{-1}$. The energy of the origin of the simulations are aligned with the most intense peak in the experimental spectrum (see text for details). The intensities of the origins in both simulations are matched with the most intense experimental peak but the baselines are set to zero counts (green trace) and the experimental 'baseline' at $77500 \mathrm{~cm}^{-1}$ (red trace). For further details, see caption to Fig. 4.

Figure 6. Stick spectrum of the largest calculated line intensities for the $\mathrm{B}^{2} \mathrm{~B}_{2}$ band of $\boldsymbol{P h C l}$, (a). The experimental PES (black trace), (b), together with a simulation (red trace) obtained by convoluting the full stick spectra with a bandwidth of $150 \mathrm{~cm}^{-1}$. The 1-photon MATI $^{11}$ (blue trace) and PIRI ${ }^{64}$ (green trace) spectra, with intensities scaled for display purposes, are also shown in (b). For further details, see caption to Fig. 4.

Figure 7. Stick spectrum of the largest calculated line intensities for the $C^{2} B_{1}$ band of $\boldsymbol{P h C l}$, (a). The experimental PES (black trace), (b), together with a simulation (red trace) obtained by convoluting the stick spectrum with a bandwidth of $300 \mathrm{~cm}^{-1}$. The baseline of the simulation is offset on the intensity scale to match the background at $93500 \mathrm{~cm}^{-1}$. For further details, see caption to Fig. 4.

Figure 8. The $1{ }^{1} \mathrm{~B}_{2}\left(\mathrm{~S}_{1}\right)$ state absorption band of $\boldsymbol{P h} \boldsymbol{C l}$. The room-temperature absorption spectrum (black trace) and a full simulation with a $20 \mathrm{~cm}^{-1}$ bandwidth (red trace), (a). The origin of the calculated simulation has been aligned with the experimental band. Bands 
induced by $v_{29}$ and $v_{28}$ are indicated by red and blue asterisks, respectively. An expansion of the origin band and its sequence band structure, together with a stick spectrum of the calculated line intensities, are shown in the inset. An expansion of the low energy region with a wavenumber shift from the electronic origin of up to $1200 \mathrm{~cm}^{-1}$ (top trace, black); a stick representation of the experimental data (middle trace, blue) in which the line positions are taken from the jet-cooled REMPI spectrum ${ }^{55}$ and the intensities are estimated from the current room-temperature absorption spectrum; a stick spectrum of the most intense non-sequence band calculated line intensities (bottom trace, black and green), (b). The bands that gain their intensity from FC overlap and HT intensity stealing are labelled in black and red, respectively, in (b).

Figure 9. Part of the background subtracted VUV absorption spectrum of $\boldsymbol{P h} \boldsymbol{C l}$. The ladders above and below the spectrum indicate [3] and [4] Rydberg states, respectively. The arrows indicate $\mathrm{IE}_{1,2}$

Figure 10. The PES of the $X^{2} B_{1}$ state, (a), the background subtracted VUV absorption spectrum of the $[1] 3 \mathrm{~d}_{3}$ state, $(\mathrm{b})$, and the $(2+1)$ REMPI spectrum of the $[1] 4 \mathrm{~d}_{12}$ state (upper ladder) and $[1] 4 \mathrm{~d}_{3}$ (lower ladder) states, (c), of $\boldsymbol{P h} \boldsymbol{C l}$. The ladders in (c) indicate $\boldsymbol{v}_{11}$ progressions starting at $v=0$.

Figure 11. The $[1] 3 \mathrm{~d} / 4 \mathrm{p}$ region of the background subtracted VUV absorption spectrum of $\boldsymbol{P h C l}$. The ladders indicate $v_{11}$ progressions $(v=0-2)$ built on the electronic origin (short legs) and one quantum of $v_{7}$ (long legs).

Figure 12. The [1]4d/4f region of the background subtracted VUV absorption, (a) and (2 + 1) REMPI, (b), spectra of $\boldsymbol{P h} \boldsymbol{C l}$. The ladders indicate $v_{11}$ progressions $(v=0-2)$ built on the electronic origin (short legs) and one quantum of $v_{7}$ (long legs). 
Figure 13. The energy region up to $\mathrm{IE}_{1}$, indicated by an arrow, of the background subtracted VUV absorption, upper trace (black), and (2+1) REMPI, lower trace (red), spectra of $\mathrm{PhCl}$.

Figure 14. The $[1] 6 \mathrm{~d} / 7 \mathrm{~d}$ region of the $(2+1)$ REMPI spectrum of $\boldsymbol{P h C l}$ recorded with linearly polarized (LP) and circularly polarized (CP) light.

Table I. The most accurate experimental ionization energies $\mathrm{IE}_{1-5}$ for $\boldsymbol{P h} \boldsymbol{C l}$ together with those calculated using UB3LYP and MCSCF methods. Valence shell numbering is used to facilitate comparison with related molecules. Our Greens' Function (GF) procedures give vertical IEs that are numerically close to experiment; calculated pole strengths are shown. Relatively small structural changes occur for each ionic state as demonstrated by the MCSCF study below.

\begin{tabular}{|c|c|c|c|c|c|c|c|}
\hline \multicolumn{2}{|c|}{$\begin{array}{c}\text { PES results } \\
\text { IE / eV }\end{array}$} & \multicolumn{2}{|c|}{$\begin{array}{c}\mathrm{GF}^{\mathrm{a}, \mathrm{b}} \\
\mathrm{IE} / \mathrm{eV}\end{array}$} & $\begin{array}{c}\text { UB3LYP }^{\mathrm{a}, \mathrm{b}} \\
\text { IE / eV }\end{array}$ & $\begin{array}{c}\mathrm{MCSCF}^{\mathrm{c}} \\
\mathrm{IE} / \mathrm{eV}\end{array}$ & $\begin{array}{c}\text { Leading } \\
\text { term }\end{array}$ & $\begin{array}{c}\text { State } \\
\text { symmetry }\end{array}$ \\
\hline Adiabatic & Vertical & Vertical & Pole strength & Adiabatic & Adiabatic & vacancy & \\
\hline $9.0722^{\mathrm{d}}$ & 9.069 & 9.070 & 0.947 & 8.861 & 8.127 & $3 b_{1}^{-1}$ & $\mathrm{X}^{2} \mathrm{~B}_{1}$ \\
\hline$<9.712^{\mathrm{e}}$ & 9.712 & 9.498 & 0.943 & 9.409 & 8.590 & $1 a_{2}^{-1}$ & $\mathrm{~A}^{2} \mathrm{~A}_{2}$ \\
\hline $11.3327^{f}$ & 11.328 & 11.942 & 0.954 & 11.053 & 11.011 & $6 b_{2}^{-1}$ & $\mathrm{~B}^{2} \mathrm{~B}_{2}$ \\
\hline 11.699 & 11.697 & 12.140 & 0.881 & - & 11.129 & $2 b_{1}^{-1}$ & $\mathrm{C}^{2} \mathrm{~B}_{1}$ \\
\hline- & 12.261 & 12.530 & 0.903 & - & 12.991 & $1 a_{1}^{-1}$ & $\mathrm{D}^{2} \mathrm{~A}_{1}$ \\
\hline
\end{tabular}

${ }^{\mathrm{a}}$ aug-cc-pVTZ X ${ }^{1} \mathrm{~A}_{1}$ ground state equilibrium structure was used.

${ }^{\mathrm{b}}$ An all valence electron study with 176 active molecular orbitals (MOs).

${ }^{\mathrm{c}}$ MCSCF 14 active MOs, 2254521 determinants, $\mathrm{X}^{1} \mathrm{~A}_{1}$ ground state equilibrium structure.

${ }^{\mathrm{d}}$ Reference 13.

${ }^{\mathrm{e}}$ See text for details.

${ }^{\mathrm{f}}$ Reference 11. 
Table II. Observed peak positions in the PES of $\boldsymbol{P h C l}$, shifts from the origin of the vibronic bands, their assignments and calculated fundamental vibrational ionic frequencies of the modes observed.

\begin{tabular}{|c|c|c|c|c|c|}
\hline $\begin{array}{c}\text { Peak } \\
\text { position/eV }\end{array}$ & $\begin{array}{l}\text { Shift from } \\
\text { origin/eV }\end{array}$ & $\begin{array}{c}\text { Peak } \\
\text { position } / \mathrm{cm}^{-1}\end{array}$ & $\begin{array}{l}\text { Shift from } \\
\text { origin } / \mathrm{cm}^{-1}\end{array}$ & $\begin{array}{l}\text { Calc. fundamental } \\
\text { frequency } / \mathrm{cm}^{-1}\end{array}$ & $\begin{array}{c}\text { Major } \\
\text { Assignment }\end{array}$ \\
\hline \multicolumn{6}{|l|}{$\mathbf{X}^{2} \mathbf{B}_{1}$} \\
\hline 8.970 & -0.98 & 72348 & -790 & & $11_{2}$ \\
\hline 9.017 & -0.51 & 72727 & -411 & 420 & $11_{1}$ \\
\hline 9.068 & 0 & 73138 & 0 & & $0^{0}$ \\
\hline 9.120 & 0.52 & 73558 & 420 & 427 & $11^{1}$ \\
\hline 9.173 & 0.105 & 73985 & 847 & & $11^{2}$ \\
\hline 9.210 & 0.142 & 74283 & 1145 & 1138,995 & $7^{1}, 9^{1}$ \\
\hline 9.260 & 0.192 & 74687 & 1539 & 1639 & $4^{1}, 7^{1} 11^{1}$ \\
\hline 9.314 & 0.246 & 75122 & 1984 & & $4^{1} 11^{1}, 7^{1} 11^{2}$ \\
\hline 9.347 & 0.279 & 75391 & 2253 & & $7^{2}$ \\
\hline 9.369 & 0.301 & 75566 & 2428 & & $4^{1} 11^{2}, 7^{1} 11^{3}$ \\
\hline 9.405 & 0.337 & 75856 & 2718 & & $4^{1} 7^{1}, 7^{2} 11^{1}$ \\
\hline 9.452 & 0.384 & 76235 & 3097 & & $4^{1} 7^{1} 11^{1}$ \\
\hline \multicolumn{6}{|l|}{$\mathbf{A}^{2} \mathbf{A}_{2}$} \\
\hline 9.712 & $0^{\mathrm{a}}$ & 78327 & $0^{\mathrm{a}}$ & & \\
\hline 9.837 & 0.125 & 79340 & 1013 & $1140,1039,995,725$ & $7^{1}, 8^{1}, 9^{1}, 10^{1}$ \\
\hline 9.969 & 0.257 & 80405 & 2078 & & \\
\hline \multicolumn{6}{|l|}{$\mathbf{B}^{2} \mathbf{B}_{2}$} \\
\hline 11.278 & -0.050 & 90960 & -406 & 420 & $11_{1}$ \\
\hline 11.328 & 0 & 91366 & 0 & & $0^{0}$ \\
\hline 11.370 & 0.042 & 91705 & 339 & 416,310 & $11^{1}, 30^{2}$ \\
\hline 11.445 & 0.117 & 92310 & 944 & 948,1003 & $7^{1}, 9^{1}$ \\
\hline 11.479 & 0.151 & 92577 & 1211 & 1201 & $6^{1}$ \\
\hline 11.488 & 0.160 & 92656 & 1290 & & $7^{1} 11^{1}$ \\
\hline 11.520 & 0.192 & 92915 & 1549 & & $6^{1} 11^{1}$ \\
\hline 11.563 & 0.235 & 93261 & 1895 & & $7^{2}$ \\
\hline \multicolumn{6}{|l|}{$\mathbf{C}^{2} \mathbf{B}_{1}$} \\
\hline 11.697 & 0 & 94336 & 0 & & $0^{0}$ \\
\hline 11.787 & 0.090 & 95062 & 726 & & \\
\hline 11.817 & 0.120 & 95304 & 968 & 998 & $9^{1}$ \\
\hline
\end{tabular}

${ }^{\mathrm{a}}$ Shift from most intense peak; see text for details. 
Table III. Experimental and calculated anharmonic frequencies for various neutral and ionic states of PhCl. The vibrational modes are numbered according to the Mulliken convention; each mode of the different states is correlated with that of the $\mathrm{X}^{1} \mathrm{~A}_{1}$ state which has the largest value in the Duschinsky matrix. All values in $\mathrm{cm}^{-1}$.

(i) Neutral states

\begin{tabular}{|c|c|c|c|c|c|}
\hline & & $\mathrm{X}^{1} \mathrm{~A}^{1}$ & & $1^{1} \mathrm{~B}_{2}$ & \\
\hline Mode & Sym. & Calc. & Exp. $^{\mathrm{a}}$ & Calc. & Exp. $^{b}$ \\
\hline 1 & \multirow[t]{11}{*}{$\mathrm{a}_{1}$} & 3104 & 3082 & 3122 & \\
\hline 2 & & 3104 & 3054 & 3112 & \\
\hline 3 & & 3029 & 3031 & 3051 & \\
\hline 4 & & 1621 & 1586 & 1554 & \\
\hline 5 & & 1503 & 1482 & 1432 & \\
\hline 6 & & 1193 & 1153 & 1166 & \\
\hline 7 & & 1109 & 1093 & 1080 & 1066 \\
\hline 8 & & 1040 & 1026 & 965 & 932 \\
\hline 9 & & 1016 & 1004 & 987 & \\
\hline 10 & & 712 & 707 & 683 & 672 \\
\hline 11 & & 420 & 417 & 384 & 377 \\
\hline 12 & \multirow[t]{3}{*}{$a_{2}$} & 963 & 962 & 681 & \\
\hline 13 & & 839 & 831 & 563 & \\
\hline 14 & & 407 & 403 & 220 & 204 \\
\hline 15 & \multirow[t]{6}{*}{$b_{1}$} & 963 & 981 & 758 & \\
\hline 16 & & 904 & 903 & 671 & \\
\hline 17 & & 741 & 741 & 572 & \\
\hline 18 & & 665 & 684 & 450 & \\
\hline 19 & & 473 & 467 & 336 & \\
\hline 20 & & 188 & 197 & 133 & 124 \\
\hline 21 & \multirow[t]{10}{*}{$\mathrm{b}_{2}$} & 3111 & 3096 & 3129 & \\
\hline 22 & & 3086 & 3067 & 3105 & \\
\hline 23 & & 1623 & 1598 & 1550 & \\
\hline 24 & & 1469 & 1447 & 1405 & \\
\hline 25 & & 1334 & 1327 & 1301 & \\
\hline 26 & & 1262 & 1272 & 1655 & \\
\hline 27 & & 1165 & 1167 & 1170 & \\
\hline 28 & & 1103 & 1068 & 1017 & $965^{\mathrm{c}}$ \\
\hline 29 & & 622 & 615 & 537 & 521 \\
\hline 30 & & 297 & 295 & 291 & 286 \\
\hline
\end{tabular}

(ii) Ionic states

\begin{tabular}{|c|c|c|c|c|c|c|c|c|c|}
\hline & & $\mathrm{X}^{2} \mathrm{~B}_{1}$ & & & & $\mathrm{~A}^{2} \mathrm{~A}_{2}$ & $\mathrm{~B}^{2} \mathrm{~B}_{2}$ & & $\mathrm{C}^{2} \mathrm{~B}_{1}$ \\
\hline Mode & Sym. & Calc. & Exp. $^{\mathrm{d}}$ & Exp. $^{\mathrm{e}}$ & Exp. $^{\mathrm{f}}$ & Calc. & Calc. & Exp. $^{\mathrm{d}}$ & Calc. \\
\hline 1 & \multirow{2}{*}{$\mathrm{a}_{1}$} & 3115 & & & & 3098 & 3122 & & 3120 \\
\cline { 1 - 7 } & & 3114 & & & & 3126 & 3116 & & 3123 \\
\hline
\end{tabular}




\begin{tabular}{|c|c|c|c|c|c|c|c|c|c|}
\hline 3 & & 3061 & & & & 3064 & 3041 & & 3060 \\
\hline 4 & & 1639 & $1593^{c}$ & & & 1580 & 1552 & & 1594 \\
\hline 5 & & 1458 & & & 1429 & 1525 & 1408 & & 1487 \\
\hline 6 & & 1214 & 1193 & 1182 & 1200 & 1204 & 1201 & & 1191 \\
\hline 7 & & 1138 & 1118 & 1106 & 1116 & 1140 & $948^{\mathrm{g}}$ & 961 & $1054^{\mathrm{g}}$ \\
\hline 8 & & 1005 & 991 & 1016 & 995 & 1039 & 1034 & & $907^{\mathrm{g}}$ \\
\hline 9 & & 995 & & 981 & 975 & 995 & 1003 & & 998 \\
\hline 10 & & 722 & 713 & 728 & 716 & 725 & 596 & $546^{\mathrm{c}}$ & 586 \\
\hline 11 & & 427 & 419 & 421 & 422 & 423 & 416 & 382 & 340 \\
\hline 12 & $\mathrm{a}_{2}$ & 1001 & & & & 1001 & 945 & & 898 \\
\hline 13 & & 805 & & & & 882 & 796 & & 776 \\
\hline 14 & & 348 & & 342 & 348 & 312 & 397 & & 355 \\
\hline 15 & $b_{1}$ & 997 & & & & 984 & 1006 & & 935 \\
\hline 16 & & 958 & & $909^{c}$ & & 890 & 908 & & $792^{\mathrm{g}}$ \\
\hline 17 & & 778 & & $812^{\mathrm{c}}$ & & 725 & 758 & & 713 \\
\hline 18 & & 596 & & $603^{c}$ & & $387^{\mathrm{g}}$ & 643 & & $480^{\mathrm{g}}$ \\
\hline 19 & & 402 & & 388 & 394 & $407^{\mathrm{g}}$ & 444 & & $363^{g}$ \\
\hline 20 & & 148 & & 165 & & 169 & 195 & & 159 \\
\hline 21 & $\mathrm{~b}_{2}$ & 3126 & & & & 3113 & 3127 & & 3128 \\
\hline 22 & & 3112 & & & & 3010 & 3088 & & 3113 \\
\hline 23 & & $1410^{\mathrm{g}}$ & $1411^{c}$ & & & 3237 & 1570 & & 1620 \\
\hline 24 & & 1524 & $1554^{c}$ & & & 1431 & 1392 & & 1485 \\
\hline 25 & & $1298^{\mathrm{g}}$ & & & & 1349 & 1323 & & 1334 \\
\hline 26 & & $1385^{\mathrm{g}}$ & & & & $1393^{\mathrm{g}}$ & 1222 & & 1271 \\
\hline 27 & & 1161 & & & & 1222 & 1163 & & 1167 \\
\hline 28 & & 1111 & & & & 1016 & 1105 & & 1115 \\
\hline 29 & & 537 & & 526 & 531 & 693 & 577 & & 609 \\
\hline 30 & & 303 & & 322 & 311 & 297 & 155 & $164^{\mathrm{c}}$ & 278 \\
\hline
\end{tabular}

${ }^{\mathrm{a}}$ Reference 54

${ }^{\mathrm{b}}$ Reference 55

${ }^{\mathrm{c}}$ Reassignment

${ }^{\mathrm{d}}$ Reference 11

${ }^{\mathrm{e}}$ Reference 56

${ }^{\mathrm{f}}$ Reference 57

${ }^{\mathrm{g}}$ Upper state normal coordinate is made up of a significant mixing of two neutral ground state normal coordinates of the same symmetry in $\mathrm{C}_{2 \mathrm{v}}$. 
Table IV. The experimental energies $\mathrm{IE}_{1-4}$ of three $\boldsymbol{P h} \boldsymbol{X}$ molecules, the separations of adjacent IEs shown in italics (all values are in $\mathrm{cm}^{-1}$ ) and estimates of the accuracy of the simulations of the vibrational profiles obtained in the current series of studies.

\begin{tabular}{|c|c|c|c|c|c|c|}
\hline & \multicolumn{2}{|c|}{$\boldsymbol{P h I}^{\mathrm{a}}$} & \multicolumn{2}{c|}{$\boldsymbol{P h B r}^{\mathrm{b}}$} & \multicolumn{2}{c|}{$\boldsymbol{P h C l}$} \\
\hline & Energy & Accuracy & Energy & Accuracy & Energy & Accuracy \\
\hline $\mathrm{IE}_{1}\left(1^{2} \mathrm{~B}_{1}\right)$ & 70638 & very good & 72570 & very good & 73172 & very good \\
\hline & 6025 & & 5294 & & 5155 & \\
\hline $\mathrm{IE}_{2}\left(1^{2} \mathrm{~A}_{2}\right)^{\mathrm{c}}$ & 76663 & good & 77864 & poor & 78327 & poor \\
\hline & 2185 & & 7958 & & 13071 & \\
\hline $\mathrm{IE}_{3}\left(1^{2} \mathrm{~B}_{2}\right)$ & 78848 & poor & 85822 & very good & 91398 & good \\
\hline & 6181 & & 4455 & & 2960 & \\
\hline $\mathrm{IE}_{4}\left(2^{2} \mathrm{~B}_{1}\right)$ & 85029 & good & 90277 & very good & 94358 & poor \\
\hline
\end{tabular}

${ }^{\mathrm{a}}$ Reference 1

${ }^{\mathrm{b}}$ Reference 3

${ }^{\mathrm{c}}$ Most intense peak in PES

Table V. Calculated adiabatic (TDDFT) excitation energies, oscillator strengths, spectral assignments and changes in $\mathrm{C}_{1}-\mathrm{Cl}$ bond lengths of some low energy excited states for $\boldsymbol{P h C l}$. Further geometric changes are shown in the supplementary material. ${ }^{29}$

\begin{tabular}{|c|c|c|c|c|c|c|}
\hline AEE/eV & AEE/cm & OS f(r) & Sym. & Leading terms & $\begin{array}{c}\text { Equilibrium } \\
\mathrm{C}_{1} \text {-Cl bond/A }\end{array}$ & $\begin{array}{c}\text { Relationship } \\
\text { to experiment }\end{array}$ \\
\hline 4.8916 & 38843 & 0.0071 & ${ }^{1} \mathrm{~B}_{2}$ & $3 \mathrm{~b}_{1} 2 \mathrm{a}_{2}{ }^{*}+1 \mathrm{a}_{2} 4 \mathrm{~b}_{1} *$ & 1.7187 & $\mathrm{~S}_{1}$ \\
\hline 5.2560 & 42380 & 0.1902 & ${ }^{1} \mathrm{~A}_{1}$ & $3 \mathrm{~b}_{1} 4 \mathrm{~b}_{1}{ }^{*}-1 \mathrm{a}_{2} 2 \mathrm{a}_{2}{ }^{*}$ & 1.7100 & $\mathrm{~S}_{2}$ \\
\hline 6.1875 & 49906 & 0.0093 & ${ }^{1} \mathrm{~B}_{1}$ & $3 \mathrm{~b}_{1} 9 \mathrm{a}_{1}{ }^{*}$ & 1.6768 & \\
\hline 6.3171 & 50947 & 0.5977 & ${ }^{1} \mathrm{~A}_{1}$ & $1 \mathrm{a}_{2} 2 \mathrm{a}_{2}{ }^{*}+3 \mathrm{~b}_{1} 4 \mathrm{~b}_{1} *$ & 1.7420 & $\mathrm{~S}_{3}$ \\
\hline 6.3186 & 50963 & 0.3109 & ${ }^{1} \mathrm{~B}_{2}$ & $1 \mathrm{a}_{2} 4 \mathrm{~b}_{1}{ }^{*}-3 \mathrm{~b}_{1} 2 \mathrm{a}_{2} *$ & 1.7288 & $\mathrm{~S}_{3}$ \\
\hline $6.5480^{\mathrm{a}}$ & 52813 & 0.0 & ${ }^{1} \mathrm{~A}_{2}$ & $1 \mathrm{a}_{2} 9 \mathrm{a}_{1}{ }^{*}$ & 1.7221 & \\
\hline
\end{tabular}

${ }^{\mathrm{a}}$ Only ${ }^{1} \mathrm{~A}_{2}$ state studied 
Table VI. Transition energies, $\mathrm{T}_{\mathrm{E}}\left(\mathrm{cm}^{-1}\right)$ and $\mathrm{n}^{*}$ values of the origin bands of the Rydberg states of $\boldsymbol{P h} \boldsymbol{C l}$ converging on the $\mathrm{X}^{2} \mathrm{~B}_{1}$ state of the ion observed in the 1-photon absorption, (1 $\left.+1^{\prime}\right)$ REMPI and $(2+1)$ REMPI spectra. ${ }^{a}$

\begin{tabular}{|c|c|c|c|c|c|c|c|c|}
\hline & $n \mathrm{p}_{1}\left(\mathrm{~A}_{1} / \mathrm{B}_{1}\right)$ & & $n \mathrm{p}_{2}\left(\mathrm{~A}_{1} / \mathrm{B}_{1}\right)$ & & $n \mathrm{p}_{3}\left(\mathrm{~A}_{2}\right)$ & & $n \mathrm{~d}_{1}\left(\mathrm{~B}_{2}\right)$ & \\
\hline$n$ & $\mathrm{~T}_{\mathrm{E}} / \mathrm{cm}^{-1}$ & $n^{*}$ & $\mathrm{~T}_{\mathrm{E}} / \mathrm{cm}^{-1}$ & $n^{*}$ & $\mathrm{~T}_{\mathrm{E}} / \mathrm{cm}^{-1}$ & $n^{*}$ & $\mathrm{~T}_{\mathrm{E}} / \mathrm{cm}^{-1}$ & $n^{*}$ \\
\hline 3 & & & & & & & $60300^{\mathrm{c}}$ & 2.92 \\
4 & $64412^{\mathrm{b}}$ & 3.54 & $64683^{\mathrm{b}}$ & 3.59 & $64690^{\mathrm{c}}$ & 3.60 & $(66080)^{\mathrm{c}}$ & 3.93 \\
\hline
\end{tabular}

\begin{tabular}{|c|c|c|c|c|c|c|c|c|c|c|}
\hline & $n \mathrm{~d}_{2}\left(\mathrm{~A}_{1}\right)$ & & $n \mathrm{~d}_{3}\left(\mathrm{~B}_{1}\right)$ & & $n \mathrm{~d}_{5}\left(\mathrm{~B}_{1}\right)$ & & $n \mathrm{f}_{1}$ & & $n \mathrm{f}_{2}$ & \\
\hline$n$ & $\mathrm{~T}_{\mathrm{E}} / \mathrm{cm}^{-1}$ & $n^{*}$ & $\mathrm{~T}_{\mathrm{E}} / \mathrm{cm}^{-1}$ & $n^{*}$ & $\mathrm{~T}_{\mathrm{E}} / \mathrm{cm}^{-1}$ & $n^{*}$ & $\mathrm{~T}_{\mathrm{E}} / \mathrm{cm}^{-1}$ & $n^{*}$ & $\mathrm{~T}_{\mathrm{E}} / \mathrm{cm}^{-1}$ & $n^{*}$ \\
\hline 3 & & & $60735^{b}$ & 2.97 & $61557^{b}$ & 3.07 & & & & \\
\hline 4 & $66004^{\mathrm{d}}$ & 3.91 & $66335^{\mathrm{b}, \mathrm{d}}$ & 4.00 & $66511^{b}$ & 4.06 & $66225^{b}$ & 3.97 & $66295^{b}$ & 3.99 \\
\hline 5 & $68698^{\mathrm{d}}$ & 4.95 & $68760^{\mathrm{d}}$ & 4.98 & & & & & & \\
\hline 6 & $70062^{\mathrm{b}, \mathrm{d}}$ & 5.94 & $70102^{\mathrm{b}, \mathrm{d}}$ & 5.97 & $70224^{b}$ & 6.10 & $70126^{b}$ & 6.00 & & \\
\hline 7 & $70896^{\mathrm{b}, \mathrm{d}}$ & 6.94 & $70928^{\mathrm{b}, \mathrm{d}}$ & 6.99 & & & $70947^{b}$ & 7.01 & & \\
\hline 8 & $71436^{\mathrm{b}, \mathrm{d}}$ & 7.94 & $71452^{\mathrm{b}, \mathrm{d}}$ & 7.98 & & & & & & \\
\hline 9 & $71808^{\mathrm{b}, \mathrm{d}}$ & 8.95 & $71838^{\mathrm{b}, \mathrm{d}}$ & 9.05 & & & & & & \\
\hline 10 & $72067^{\mathrm{b}, \mathrm{d}}$ & 9.94 & $72072^{\mathrm{b}, \mathrm{d}}$ & 9.97 & & & & & & \\
\hline 11 & $72263^{\mathrm{b}, \mathrm{d}}$ & 10.96 & $72254^{\mathrm{b}, \mathrm{d}}$ & 10.90 & & & & & & \\
\hline 12 & $72411^{\mathrm{b}, \mathrm{d}}$ & 11.97 & & & & & & & & \\
\hline 13 & $72526^{\mathrm{b}, \mathrm{d}}$ & 12.98 & & & & & & & & \\
\hline 14 & $72614^{\mathrm{d}}$ & 13.96 & & & & & & & & \\
\hline 15 & $72683^{d}$ & 14.90 & & & & & & & & \\
\hline 16 & $72745^{\mathrm{d}}$ & 15.94 & & & & & & & & \\
\hline \multicolumn{11}{|c|}{${ }^{\mathrm{a}} n^{*}$ values calculated using $\mathrm{IE}_{1}$ of $73172 \mathrm{~cm}^{-1}{ }^{13}$} \\
\hline
\end{tabular}


Table VII. Transition energies, $\mathrm{T}_{\mathrm{E}}\left(\mathrm{cm}^{-1}\right)$ and $\mathrm{n}^{*}$ values of the origin bands of the Rydberg states of $\boldsymbol{P h C l}$ converging on the $\mathrm{B}^{2} \mathrm{~B}_{2}$ state of the ion observed in the 1-photon absorption spectrum. $^{\text {a }}$

\begin{tabular}{rl|c|c|c|c|c|c|c|c|c|c|}
\hline & $n \mathrm{~s}$ & & $n \mathrm{p}_{1}$ & & $n \mathrm{p}_{2}$ & & $n \mathrm{~d}_{1}$ & & $n \mathrm{~d}_{2}$ & \\
\hline$n$ & $\mathrm{~T}_{\mathrm{E}} / \mathrm{cm}^{-1}$ & $n^{*}$ & $\mathrm{~T}_{\mathrm{E}} / \mathrm{cm}^{-1}$ & $n^{*}$ & $\mathrm{~T}_{\mathrm{E}} / \mathrm{cm}^{-1}$ & $n^{*}$ & $\mathrm{~T}_{\mathrm{E}} / \mathrm{cm}^{-1}$ & $n^{*}$ & $\mathrm{~T}_{\mathrm{E}} / \mathrm{cm}^{-1}$ & $n^{*}$ \\
\hline 3 & 68611 & 2.19 & 73314 & 2.46 & 74239 & 2.53 & 78064 & 2.87 & 79872 & 3.08 \\
4 & 81103 & 3.26 & 82645 & 3.54 & 83160 & 3.65 & 84140 & 3.89 & 84926 & 4.12 \\
5 & 85397 & 4.27 & 86133 & 4.56 & 86430 & 4.70 & 86843 & 4.91 & & \\
\hline
\end{tabular}

Table VIII. Transition energies, $\mathrm{T}_{\mathrm{E}}\left(\mathrm{cm}^{-1}\right)$ and $\mathrm{n}^{*}$ values of the origin bands of the Rydberg states of $\boldsymbol{P h} \boldsymbol{C l}$ converging on the $\mathrm{C}^{2} \mathrm{~B}_{1}$ state of the ion observed in the 1-photon absorption spectrum. $^{\text {a }}$

\begin{tabular}{|l|c|c|c|c|c|c|c|c|}
\hline & $n \mathrm{~s}$ & & $n \mathrm{p}$ & & $n \mathrm{~d}_{1}$ & & $n \mathrm{~d}_{2}$ & \\
\hline$n$ & $\mathrm{~T}_{\mathrm{E}} / \mathrm{cm}^{-1}$ & $n^{*}$ & $\mathrm{~T}_{\mathrm{E}} / \mathrm{cm}^{-1}$ & $n^{*}$ & $\mathrm{~T}_{\mathrm{E}} / \mathrm{cm}^{-1}$ & $n^{*}$ & $\mathrm{~T}_{\mathrm{E}} / \mathrm{cm}^{-1}$ & $n^{*}$ \\
\hline 3 & 71400 & 2.19 & 76864 & 2.50 & 82068 & 2.99 & 83542 & 3.19 \\
4 & 84140 & 3.28 & & & & & & \\
\hline
\end{tabular}

